\title{
Predicting pattern formation in particle interactions
}

\author{
James von Brecht, David Uminsky*, \\ Theodore Kolokolnikov; Andrea Bertozzi*
}

May 20, 2011

\begin{abstract}
Large systems of particles interacting pairwise in $d$-dimensions give rise to extraordinarily rich patterns. These patterns generally occur in two types. On one hand, the particles may concentrate on a co-dimension one manifold such as a sphere (in 3D) or a ring (in 2D). Localized, space-filling, codimension zero patterns can occur as well. In this paper, we utilize a dynamical systems approach to predict such behaviors in a given system of particles. More specifically, we develop a non-local linear stability analysis for particles uniformly distributed on a $d-1$ sphere. Remarkably, the linear theory accurately characterizes the patterns in the ground states from the instabilities in the pairwise potential. This aspect of the theory then allows us to address the issue of inverse statistical mechanics in selfassembly: given a ground state exhibiting certain instabilities, we construct a potential that corresponds to such a pattern.
\end{abstract}

\section{Introduction}

The mathematics of interacting particles pervades many disciplines, from physics and biology to control theory and engineering. Classical examples from physics and chemistry range from the distribution of electrons in the Thomson problem, to VSEPR theory, self-assembly processes, and protein folding. In biology, similar mathematical models help explain the complex phenomena observed in locust swarms and bacterial colonies. In engineering, particle models have been successfully used in many areas of cooperative control, including applications to robotic swarming. In each of these models, the collective behavior is confined near the center of mass of the particles. This can be imposed artificially, as in the Thomson problem, or can result due to the properties of the interaction potential itself. Moreover, different confining potentials may give rise to densities that concentrate on a co-dimension one manifold, or form localized, fully co-dimension zero structures. In particular, the effect of differences in the confining potentials remains evident in passing to the continuum limit. In this paper, we develop a method to predict features of the resulting patterns from properties of the potential, and vice-versa.

An understanding of co-dimension one ground states is germane to many applications. For instance, in a discrete setting such states arise in both point vortex theory [26, 25, 16, 2 as well as the Thomson problem 28, 1, 40, 8, 9. In the context of point vortex theory, vortices restricted to a sphere can organize into both platonic solid and ring configurations [26, 25, 16. Similar spherical configurations also arise in the classical Thomson's problem, which asks for the lowest potential energy configuration of $N$ repelling electrons fixed to said surface. For small numbers of electrons, the minimizers exhibit platonic solid configurations. As the number of electrons increases, a wide variety of spherical lattices may form, including non-platonic solids as well as lattices with higher order defects. Complex, co-dimension zero patterns also arise in biology, and have inspired researchers to develop mathematical models that can help explain, both evolutionarily and biologically, why and how these self-assembled patterns form [6, 29, 27, 17, 24, 12, 15, 22]. Such models have proven fruitful in modeling locust swarms [3, 21, 36, where the techniques capture the unique swarm shapes of locusts. These models also help explain rings, annuli, and other complex, spotted patterns in bacterial

${ }^{*}$ UCLA Dept. of Mathematics, Box 951555, Los Angeles, CA 90095-1555

${ }^{\dagger}$ Department of Mathematics and Statistics, Dalhousie University, Halifax, Canada 
colonies that form under stress in the lab [38, 10, 18, 4. Many of these same models have been exploited in the area of cooperative control [4] and boundary tracking algorithms for autonomous, flocking robots 7 .

We formulate ground state patterns as extrema of an $N$-particle pairwise interaction energy

$$
E\left(\mathbf{x}_{1}, \ldots, \mathbf{x}_{N}\right)=\sum_{i, j \neq i} P\left(\left|\mathbf{x}_{i}-\mathbf{x}_{j}\right|\right):=\sum_{i, j \neq i} V\left(\frac{1}{2}\left|\mathbf{x}_{i}-\mathbf{x}_{j}\right|^{2}\right),
$$

where $P(\sqrt{2 s}):=V(s)$ denotes a repulsive-attractive potential, i.e. decreasing for all $s<s_{0}$ and increasing for all $s>s_{0}$. To compute local minimizers, we associate a gradient flow to the interaction energy (1)

$$
\frac{\mathrm{d} \mathbf{x}_{i}}{\mathrm{~d} t}=-\nabla_{\mathbf{x}_{i}} E=\frac{1}{N} \sum_{\substack{j=1 \ldots N \\ j \neq i}} g\left(\frac{1}{2}\left|\mathbf{x}_{i}-\mathbf{x}_{j}\right|^{2}\right)\left(\mathbf{x}_{i}-\mathbf{x}_{j}\right), \quad i=1 \ldots N
$$

where $g(s)=-V_{s}(s)$ gives the force. We shall call a sequence of $N$-particle minimizers

$$
\left\{\mathbf{x}_{i}\right\}_{i=1}^{N}=\arg \min _{\mathbf{y}_{1}, \ldots, \mathbf{y}_{N}} E\left(\mathbf{y}_{1}, \ldots, \mathbf{y}_{N}\right)
$$

confined if they remain uniformly bounded in space as a function of the number of particles. In such cases, in the large $N$ limit, the minimizers permit a consistent continuum description in terms of a density of particles restricted to a bounded region of space. As the number of particles increases, the resulting ground state converges to this continuum description in the sense of probability measures. Moreover, variations in potentials give rise to different minimizers, so differences in the potentials remain evident as $N \rightarrow \infty$. This stands in contrast to non-confined minimizers, which do not remain bounded. Without confinement, we cannot rely on a density description, nor can we necessarily distinguish differences in potentials as $N \rightarrow \infty$. We shall call a potential confining if all minimizers are confined, and non-confining otherwise.

A given class of potentials may yield both types of behavior. For example, inverse power law potentials

$$
g(s)=V^{\prime}(s)=s^{-p}-s^{-q}
$$

can have both confined and non-confined minimizers, depending on the parameters $p$ and $q$. Choosing $(p, q)=(7,4)$, i.e a Lennard-Jones interaction kernel, yields a sequence of global minimizers which converge to the zero density state. On the other hand, as we show in $\S 6$ the choice $(p, q)=\left(\frac{1}{3}, \frac{1}{6}\right)$ yields a sequence of minimizers that converge to the uniform measure on a 2 -sphere of fixed radius $R=2^{-1 / 2}\left(\frac{11}{10}\right)^{3}$. The repulsive-attractive Morse potential

$$
V(s)=\mathrm{e}^{-\sqrt{2 s}}-F \mathrm{e}^{-L \sqrt{2 s}}
$$

also exhibits this dichotomy as $(F, L)$ vary [21, 11]. In particular, we can not distinguish between these two classes of potentials from a large $N$ limit of their ground states when the minimizers are not confined.

In contrast, the configurations in figure 1 result from a two parameter family of confining potentials. We numerically integrate 22 to steady-state with the regularized step function from [19]

$$
g(s)=\frac{\tanh (a(1-\sqrt{2 s}))+b}{\sqrt{2 s}}, \quad 0<a, \quad-\tanh (a)<b<1,
$$

as the interaction kernel. Simply by controlling the amount of local repulsion at the origin $(b)$ and the slope of the transition between repulsion and attraction $(a)$, many types of patterns emerge, and these differences persist as $N \rightarrow \infty$. For instance, a uniform distribution of particles on a sphere often minimizes (1). Properties such as the radius of the sphere change as the parameters $(a, b)$ vary, and predicting the radius from a continuum approximation proves increasingly accurate as the number of particles grows. Therefore, unlike the non-confining Morse and inverse power potentials, we can hope to discern differences in parameter values, and in particular, explain the transition from spherical solutions to distributions characterized by higher symmetry and full three dimensionality using a continuum approach.

To better understand this phenomenon, we develop a continuum formulation of (2) to study the stability of a uniform sphere $\mathcal{S}^{d-1}$. We formally derive this continuum formulation of 2 in $\$ 2$, so that we may describe a pairwise interaction of particles lying on a co-dimension one surface. Using this formulation, we 


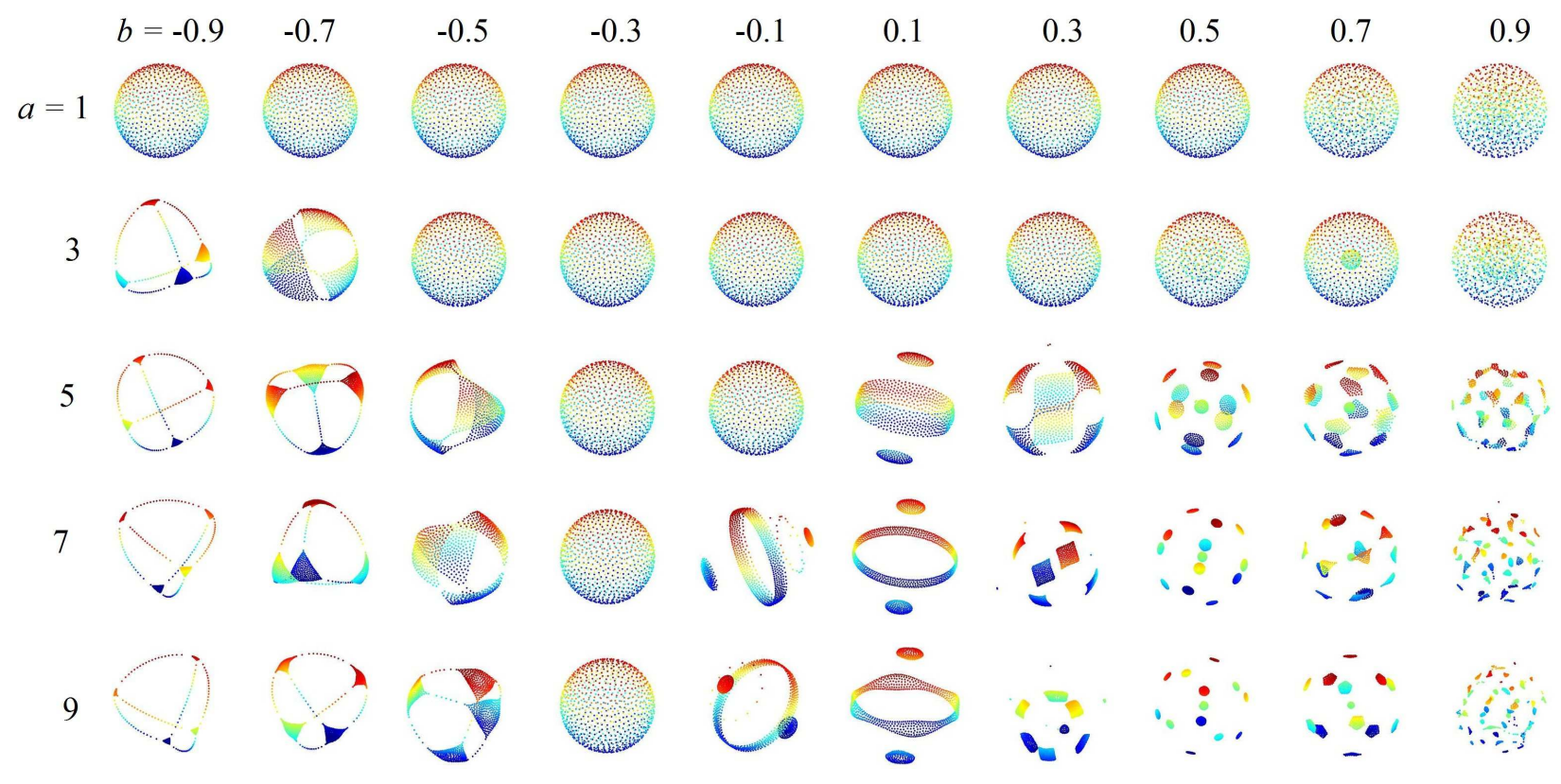

Figure 1: Top: Minimizers of the energy (1) with force law (4).

derive our main result in $\$ 3$ that the eigenvalue problem associated to the 2 -sphere of radius $R$ reduces to the decoupled series of $2 \times 2$ scalar problems given by equation (30). Each eigenvalue problem determines a solution to the linearized equations in terms of spherical harmonics. We use this characterization to predict how instabilities perturb the density away from uniform in $\S \S 3.2$. We complete the eigenvalue problem for arbitrary dimensions $d \geq 2$ in $\& 4$ Remarkably, the eigenvalue problem remains $2 \times 2$ and scalar independently of the dimension of space. Moreover, our analysis depends only on values of the potential and its derivatives on $\left[0,2 R^{2}\right]$. Thus, once we know the length scale of the radius our analysis applies regardless of the far-field behavior of the potential. In $\$ 5$ we derive asymptotic expressions for the eigenvalues in theorem 5.1 As a first corollary we establish the linear well-posedness of uniformly distributed sphere solutions, which serves as the analogue in our context of the classic Kelvin-Helmholtz instability for vortex sheets [20, 23, 34. In a second corollary, we consider potentials $V$ with the form

$$
-V_{s}(s):=g(s)=\sum_{i=1}^{\infty} c_{i} s^{p_{i}}
$$

where $p_{i}<p_{i+1}$ and $c_{1}>0$ to ensure an interaction kernel with repulsion in the short-range. We show that only finitely many unstable modes exist precisely when

$$
\text { (i) } \int_{-1}^{1} g\left(R^{2}(1-s)\right) d s+4 g\left(2 R^{2}\right)<0 \quad \text { and } \quad \text { (ii) } p_{1} \in\left(-\frac{d-1}{2}, 0\right) \cup \bigcup_{n=0}^{\infty}(2 n+1,2 n+2) \text {. }
$$

In this case, we can predict complex patterns in the resulting ground state from the unstable spherical harmonics. These conditions also allow us to predict the co-dimension of the ground state. We highlight these aspects of the theory in $\$ 6$ with several examples. This method also offers us a way to directly construct a potential with a specified instability which is related to the classical inverse statistical mechanics problem [9, 31, 30, 37, 13, 39. We follow with a brief summary. 


\section{Pairwise Interactions on a Surface}

We begin by formally deriving the relevant equations to describe a continuum of pairwise-interacting particles on a surface. For this, we consider the active scalar equations in three dimensions

$$
\begin{array}{r}
\rho_{t}(\mathbf{x}, t)+\nabla \cdot(\rho(\mathbf{x}, t) \mathbf{u}(\mathbf{x}, t))=0, \quad \mathbf{x} \in \mathbb{R}^{3}, t \geq 0 \\
\mathbf{u}(\mathbf{x}, t)=\int_{\mathbb{R}^{3}} g\left(\frac{1}{2}|\mathbf{x}-\mathbf{y}|^{2}\right)(\mathbf{x}-\mathbf{y}) \rho(\mathbf{y}, t) \mathrm{d} \mathbf{y},
\end{array}
$$

where $\rho$ describes the density of particles, the kernel $g$ describes the interaction of particles, and $\mathbf{u}$ describes the velocity of a particular particle due to the interaction. After constraining the density of particles to lie on a dynamically evolving surface $\mathbf{X}(\xi, \eta, t)$, where $(\xi, \eta)$ lie in a Lagrangian coordinate domain $D \subset \mathbb{R}^{2}$, the density defines a distribution of the form

$$
\rho(\mathbf{x}, t):=\int_{D} \delta(\mathbf{x}-\mathbf{X}(\xi, \eta, t)) f(\xi, \eta, t) \mathrm{d} \xi \mathrm{d} \eta .
$$

In other words, $\langle\rho, \phi\rangle=\int_{0}^{\infty} \int_{D} \phi(\mathbf{X}(\xi, \eta, t)) f(\xi, \eta, t) \mathrm{d} \xi \mathrm{d} \eta \mathrm{d} t$ holds for all test functions $\phi \in C_{0}^{\infty}\left(\mathbb{R}^{3} \times\{t \geq 0\}\right)$. With a density in this form, we say (8) defines a solution to (7) in the sense of distributions. We therefore specify equations for $\mathbf{X}$ and $f$ so that

$$
\begin{array}{r}
\int_{0}^{\infty} \int_{D}\left(\phi_{t}+\mathbf{u} \cdot \nabla \phi\right)(\mathbf{X}(\xi, \eta, t), t) f(\xi, \eta, t) \mathrm{d} \xi \mathrm{d} \eta \mathrm{d} t=0 \\
\mathbf{u}=\int_{D} g\left(\frac{1}{2}|\mathbf{x}-\mathbf{X}(\xi, \eta, t)|^{2}\right)(\mathbf{x}-\mathbf{X}(\xi, \eta, t)) f(\xi, \eta, t) \mathrm{d} \xi \mathrm{d} \eta
\end{array}
$$

for all $\phi \in C_{0}^{\infty}$.

Addressing first the motion of the surface, as $\mathbf{X}(\xi, \eta, t)$ represents the position of the particle with label $(\xi, \eta)$, each point on the surface evolves according to the velocity field $\mathbf{u}$ at that point, so that

$$
\begin{gathered}
\frac{\partial \mathbf{X}}{\partial t}(\xi, \eta, t)=\mathbf{u}(\mathbf{X}(\xi, \eta, t), t)= \\
\int_{D} g\left(\frac{1}{2}\left|\mathbf{X}(\xi, \eta, t)-\mathbf{X}\left(\xi^{\prime}, \eta^{\prime}, t\right)\right|^{2}\right)\left(\mathbf{X}(\xi, \eta, t)-\mathbf{X}\left(\xi^{\prime}, \eta^{\prime}, t\right)\right) f\left(\xi^{\prime}, \eta^{\prime}, t\right) \mathrm{d} \xi^{\prime} \mathrm{d} \eta^{\prime} .
\end{gathered}
$$

Combining (9) and (11) with the fact that $\frac{\partial}{\partial t}\{\phi(\mathbf{X}, t)\}=\left(\phi_{t}+\frac{\partial \mathbf{X}}{\partial t} \cdot \nabla \phi\right)(\mathbf{X}, t)$, we discover $f$ must satisfy $\int_{0}^{\infty} \int_{D} \frac{\partial}{\partial t}\{\phi(\mathbf{X}, t)\} f(\xi, \eta, t) \mathrm{d} \xi \mathrm{d} \eta \mathrm{d} t=0$. Integrating by parts in time, this gives $\int_{0}^{\infty} \int_{D} \phi(\mathbf{X}, t) f(\xi, \eta, t)_{t} \mathrm{~d} \xi \mathrm{d} \eta \mathrm{d} t=$ 0 for all $\phi$, whence $f(\xi, \eta, t) \equiv f(\xi, \eta, 0)$. Therefore, given an initial density

$$
\rho(\mathbf{x}, 0)=\rho_{0}(\mathbf{x})=\int_{D} \delta\left(\mathbf{x}-\mathbf{X}_{0}(\xi, \eta)\right) f_{0}(\xi, \eta) \mathrm{d} \xi \mathrm{d} \eta
$$

by evolving the surface according to

$$
\begin{aligned}
\mathbf{X}_{t} & =\int_{D} g\left(\frac{1}{2}\left|\mathbf{X}-\mathbf{X}^{\prime}\right|^{2}\right)\left(\mathbf{X}-\mathbf{X}^{\prime}\right) f_{0}\left(\xi^{\prime}, \eta^{\prime}\right) \mathrm{d} \xi^{\prime} \mathrm{d} \eta^{\prime} \\
\mathbf{X} & =\mathbf{X}(\xi, \eta, t) \quad \mathbf{X}^{\prime}=\mathbf{X}\left(\xi^{\prime}, \eta^{\prime}, t\right) \quad \mathbf{X}(\xi, \eta, 0)=\mathbf{X}_{0}(\xi, \eta)
\end{aligned}
$$

we obtain a distribution solution to (7).

Remark 2.1. Equations (12) easily generalize to any dimension. We use the general d-dimensional form in 8

Although it is superfluous for determining the evolution of the surface, it will prove useful at times to re-write (8) in a more conventional form,

$$
\rho(\mathbf{x}, t):=\int_{D} \delta(\mathbf{x}-\mathbf{X}) \rho_{S}(\xi, \eta, t)\left|\mathbf{X}_{\xi} \times \mathbf{X}_{\eta}\right|(t) \mathrm{d} \xi \mathrm{d} \eta
$$


The auxiliary quantity $\rho_{S}(\xi, \eta, t)$ then has the natural interpretation as the density of particles along the surface. The requirement that $\frac{\partial f}{\partial t}=0$ implies the density evolves according to $\frac{\partial \rho_{S}}{\partial t}=-\rho_{S} \frac{\left|\mathbf{X}_{\xi} \times \mathbf{X}_{\eta}\right|_{t}}{\left|\mathbf{X}_{\xi} \times \mathbf{X}_{\eta}\right|}$, which when coupled to $(12)$ becomes the extension to three dimensions of the corresponding equations from [34] for curves in two dimensions. In this manner, the evolution of the surface alone determines the density of particles along it; the surface $\mathbf{X}(\xi, \eta)$ is fundamental and the density is derived. For this reason, we focus our analysis on $\mathbf{X}$, and use this to determine properties of $\rho_{S}$.

\section{Eigenvalue Problem in Three Dimensions}

We now determine when a sphere of uniform density defines a linearly stable solution to (12). In $\S ₫ 3.1$, we linearize (12) about a uniform sphere, and then reduce the problem to a decoupled series of scalar eigenvalue problems involving a single spherical harmonic. We proceed with the calculations in a manner that makes the appearance of spherical harmonics self-evident, as the ideas behind the calculation itself prove useful for other problems. The existence of uniform sphere solutions follows as an easy application, for instance, so we postpone it until after we derive the eigenvalue problem. In $\S \S 3.2$, we use knowledge of the eigenvalue problem to linearize the density $\rho_{S}$ about the uniform distribution on the sphere. This later proves useful for interpreting our stability conditions in $\S 5$, and also in $\S 6$ for comparing our analysis against numerics.

\subsection{Linearization of the Surface}

We begin by considering the evolution equations for a surface 12 for the particular instance of $f_{0}$ which yields a sphere of radius $R$ and uniform density as steady-state,

$$
\mathbf{X}_{t}=\int_{-\pi}^{\pi} \int_{0}^{\pi} g\left(\frac{1}{2}\left|\mathbf{X}-\mathbf{X}^{\prime}\right|^{2}\right)\left(\mathbf{X}-\mathbf{X}^{\prime}\right) \sin \eta^{\prime} \mathrm{d} \eta^{\prime} \mathrm{d} \xi^{\prime}
$$

Here, we parameterize a sphere of radius $R$ as $\mathbf{X}(\xi, \eta)=\Theta_{1}(\xi) \Theta_{2}(\eta) R \mathbf{e}_{1}$ for $-\pi \leq \xi \leq \pi$ and $0 \leq \eta \leq \pi$. The $3 \times 3$ matrix $\Theta_{1}$ represents rotation in the $y$ - $z$ plane, $\Theta_{2}$ rotates in the $x$ - $y$ plane, and of course $\mathbf{e}_{1}=(1,0,0)^{t}$.

Write a perturbation $\delta \mathbf{X}$ of the steady-state in the form

$$
\delta \mathbf{X}=\Theta_{1}(\xi) \Theta_{2}(\eta)\left(R \mathbf{e}_{1}+\epsilon(\xi, \eta) e^{\lambda t}\right),
$$

with the goal of choosing the ansatz for $\epsilon \in \mathbb{R}^{3}$ in such a way that the linear equations for $\epsilon$ reduce to a scalar eigenvalue problem for $\lambda$ and scalar coefficients that will determine $\epsilon$. First, we substitute $\delta \mathbf{X}$ into 13) and obtain

$$
\lambda \Theta_{1}(\xi) \Theta_{2}(\eta) \epsilon(\xi, \eta)=\int_{-\pi}^{\pi} \int_{0}^{\pi} g\left(\frac{1}{2}\left|\delta \mathbf{X}-\delta \mathbf{X}^{\prime}\right|^{2}\right)\left(\delta \mathbf{X}-\delta \mathbf{X}^{\prime}\right) \sin \eta^{\prime} \mathrm{d} \eta^{\prime} \mathrm{d} \xi^{\prime}
$$

Decomposing $\delta \mathbf{X}-\delta \mathbf{X}^{\prime}:=\mathbf{X}_{1}+\mathbf{X}_{2}$, where $\mathbf{X}_{1}=\left[\Theta_{1}(\xi) \Theta_{2}(\eta)-\Theta_{1}\left(\xi^{\prime}\right) \Theta_{2}\left(\eta^{\prime}\right)\right] R \mathbf{e}_{1}$ and $\mathbf{X}_{2}=\Theta_{1}(\xi) \Theta_{2}(\eta) \epsilon(\xi, \eta)-$ $\Theta_{1}\left(\xi^{\prime}\right) \Theta_{2}\left(\eta^{\prime}\right) \epsilon\left(\xi^{\prime}, \eta^{\prime}\right)$, we expand to first order in $\mathbf{X}_{2}$ and use the fact that the sphere is a steady-state to obtain

$$
\begin{gathered}
\lambda \Theta_{1}(\xi) \Theta_{2}(\eta) \epsilon(\xi, \eta)= \\
\int_{-\pi}^{\pi} \int_{0}^{\pi}\left\{g\left(\frac{1}{2}\left|\mathbf{X}_{1}\right|^{2}\right) \mathbf{X}_{2}+g_{s}\left(\frac{1}{2}\left|\mathbf{X}_{1}\right|^{2}\right)\left(\mathbf{X}_{1} \cdot \mathbf{X}_{2}\right) \mathbf{X}_{1}\right\} \sin \eta^{\prime} \mathrm{d} \eta^{\prime} \mathrm{d} \xi^{\prime}
\end{gathered}
$$

Denoting by $M$ the matrix $M:=\Theta_{2}^{-1}(\eta) \Theta_{1}\left(\xi^{\prime}-\xi\right) \Theta_{2}\left(\eta^{\prime}\right)$ and by $I$ the $3 \times 3$ identity matrix, simple calculations yield

$$
\begin{gathered}
\Theta_{2}^{-1}(\eta) \Theta_{1}^{-1}(\xi) \mathbf{X}_{1}=(I-M) R \mathbf{e}_{1}, \quad \Theta_{2}^{-1}(\eta) \Theta_{1}^{-1}(\xi) \mathbf{X}_{2}=\epsilon(\xi, \eta)-M \epsilon\left(\xi^{\prime}, \eta^{\prime}\right) \\
\mathbf{X}_{1} \cdot \mathbf{X}_{2}=(I-M) R \mathbf{e}_{1} \cdot \epsilon(\xi, \eta)+\left(I-M^{t}\right) R \mathbf{e}_{1} \cdot \epsilon\left(\xi^{\prime}, \eta^{\prime}\right), \quad\left|\mathbf{X}_{1}\right|=\left|(I-M) R \mathbf{e}_{1}\right| .
\end{gathered}
$$


By premultiplying 16 with $\Theta_{2}^{-1} \Theta_{1}^{-1}$ and separating terms involving $\epsilon(\xi, \eta)$ from terms involving $\epsilon\left(\xi^{\prime}, \eta^{\prime}\right)$, we obtain the linearized problem

$$
\begin{array}{r}
\lambda \epsilon(\xi, \eta)=\int_{-\pi}^{\pi} \int_{0}^{\pi}\left\{g\left(\frac{1}{2}|\mathbf{v}|^{2}\right) I+g_{s}\left(\frac{1}{2}|\mathbf{v}|^{2}\right) \mathbf{v} \otimes \mathbf{v}\right\} \epsilon(\xi, \eta) \sin \eta^{\prime} \mathrm{d} \eta^{\prime} \mathrm{d} \xi^{\prime}+ \\
\int_{-\pi}^{\pi} \int_{0}^{\pi}\left\{g_{s}\left(\frac{1}{2}|\mathbf{v}|^{2}\right) \mathbf{v} \otimes \underline{\underline{\mathbf{v}}}-g\left(\frac{1}{2}|\mathbf{v}|^{2}\right) M\right\} \epsilon\left(\xi^{\prime}, \eta^{\prime}\right) \sin \eta^{\prime} \mathrm{d} \eta^{\prime} \mathrm{d} \xi^{\prime},
\end{array}
$$

where we define $\mathbf{v}:=(I-M) R \mathbf{e}_{1}$ and $\underline{\underline{\mathbf{v}}}:=\left(I-M^{t}\right) R \mathbf{e}_{1}$.

The difficulty now lies in choosing $\bar{\epsilon}(\xi, \eta)$ in such a way that the continuous eigenvalue problem (17) reduces to a simple scalar eigenvalue problem. To find the way forward, we recall the analogous situation in two dimensions, as detailed in [19. In that setting, the continuous eigenvalue problem reads

$$
\begin{aligned}
\lambda \epsilon(s) & =\int_{-\pi}^{\pi}\left\{g\left(\frac{1}{2}|\mathbf{v}|^{2}\right) I+g_{s}\left(\frac{1}{2}|\mathbf{v}|^{2}\right) \mathbf{v} \otimes \mathbf{v}\right\} \epsilon(s) \mathrm{d} s^{\prime}+ \\
& \int_{-\pi}^{\pi}\left\{g_{s}\left(\frac{1}{2}|\mathbf{v}|^{2}\right) \mathbf{v} \otimes \underline{\underline{\mathbf{v}}}-g\left(\frac{1}{2}|\mathbf{v}|^{2}\right) \Theta\left(s^{\prime}-s\right)\right\} \epsilon\left(s^{\prime}\right) \mathrm{d} s^{\prime}
\end{aligned}
$$

where $\mathbf{v}=\left(I-\Theta\left(s^{\prime}-s\right)\right) R \mathbf{e}_{1}, \underline{\underline{\mathbf{v}}}=\left(I-\Theta\left(s-s^{\prime}\right)\right) R \mathbf{e}_{1}$ and $\Theta(s)$ denotes a $2 \times 2$ rotation matrix. We can write this as

$$
\lambda \epsilon(s)=\int_{-\pi}^{\pi} M_{1}\left(s-s^{\prime}\right) \epsilon(s) \mathrm{d} s^{\prime}+\int_{-\pi}^{\pi} M_{2}\left(s-s^{\prime}\right) \epsilon\left(s^{\prime}\right) \mathrm{d} s^{\prime},
$$

for some $2 \times 2$ matrices $M_{i}$. Letting $M_{i}^{j k}$ denote the $(j, k)$ entry of the matrix $M_{i}$, we find that both matrices possess even, periodic entries in $s-s^{\prime}$ whenever $j=k$, and odd, periodic entries whenever $j \neq k$. Changing variables (i.e. reparameterizing the circle) in the first integral, we have

$$
\int_{-\pi}^{\pi} M_{i}^{j k}\left(s-s^{\prime}\right) \mathrm{d} s^{\prime}=\int_{-\pi}^{\pi} M_{i}^{j k}(\theta) \mathrm{d} \theta \propto \delta_{j k}
$$

Thus, the first term on the RHS of (18) simplifies to a constant diagonal matrix times $\epsilon(s)$. We then substitute the known ansatz for $\epsilon$ from [19] into the second integral, $\epsilon\left(s^{\prime}\right)=\left(c_{1} \cos \left(m s^{\prime}\right), c_{2} \sin \left(m s^{\prime}\right)\right)^{t}$ for some constants $c_{1}$ and $c_{2}$, change variables and simplify. Along the first column of $M_{2}$, we find

$$
\begin{aligned}
& \int_{-\pi}^{\pi} M_{2}^{11}\left(s-s^{\prime}\right) c_{1} \cos \left(m s^{\prime}\right) \mathrm{d} s^{\prime}=\int_{-\pi}^{\pi} M_{2}^{11}(\theta) c_{1} \cos (m \theta+m s) \mathrm{d} \theta \propto \cos (m s) \\
& \int_{-\pi}^{\pi} M_{2}^{21}\left(s-s^{\prime}\right) c_{1} \cos \left(m s^{\prime}\right) \mathrm{d} s^{\prime}=\int_{-\pi}^{\pi} M_{2}^{21}(\theta) c_{2} \cos (m \theta+m s) \mathrm{d} \theta \propto \sin (m s)
\end{aligned}
$$

due to the even-odd structure of $M_{2}$. Arguing similarly along the second column, the second term on the RHS of (18) simplifies as

$$
\int_{-\pi}^{\pi} M_{2}\left(s-s^{\prime}\right) \epsilon\left(s^{\prime}\right) \mathrm{d} s^{\prime}=D\left(c_{1}, c_{2}, m\right)(\cos (m s), \sin (m s))^{t},
$$

where $D$ denotes a constant, diagonal matrix depending upon $c_{1}, c_{2}$ and the Fourier coefficients of the entries of $M_{2}$. Moreover, $D\left(c_{1}, c_{2}, m\right)$ is linear in the coefficients $\left(c_{1}, c_{2}\right)$ that determine $\epsilon$. As the first integral also results in something of this form, the continuous problem reduces to a scalar eigenvalue problem in $\left(c_{1}, c_{2}\right)$. From (20), then, we deduce the essential property of the ansatz: $\int_{-\pi}^{\pi} M_{2}^{i j}\left(s-s^{\prime}\right) \epsilon_{j}\left(s^{\prime}\right) \mathrm{d} s^{\prime} \propto \epsilon_{i}(s)$.

Returning now to the three-dimensional case, regardless of the choice of the ansatz $\epsilon(\xi, \eta)$, we first must show the first integral in (17) yields a constant, diagonal matrix. To do this, note the integrand depends only upon the vector $\mathbf{v}$. Looking at the definition of $\mathbf{v}$, for fixed $(\xi, \eta)$ and for $-\pi \leq \xi^{\prime} \leq \pi, 0 \leq \eta^{\prime} \leq \pi$, we see that $\mathbf{v}$ simply represents a parameterization of $\partial B\left(R \mathbf{e}_{1}, R\right)$, i.e. the sphere of radius $R$ centered at the point $(R, 0,0)^{t}$. Moreover, $\left|\mathbf{v}_{\xi^{\prime}} \times \mathbf{v}_{\eta^{\prime}}\right|=\sin \eta^{\prime}$, so that by definition

$$
\int_{-\pi}^{\pi} \int_{0}^{\pi}\left\{g\left(\frac{1}{2}|\mathbf{v}|^{2}\right) I+g_{s}\left(\frac{1}{2}|\mathbf{v}|^{2}\right) \mathbf{v} \otimes \mathbf{v}\right\} \epsilon(\xi, \eta) \sin \eta^{\prime} \mathrm{d} \eta^{\prime} \mathrm{d} \xi^{\prime}=
$$




$$
\left(\int_{\partial B\left(R \mathbf{e}_{1}, R\right)} G(\mathbf{x}) \mathrm{d} S(\mathbf{x})\right) \epsilon(\xi, \eta),
$$

where the $3 \times 3$ matrix valued function $G(\mathbf{x})=g\left(\frac{1}{2}|\mathbf{x}|^{2}\right) I+g_{s}\left(\frac{1}{2}|\mathbf{x}|^{2}\right) \mathbf{x} \otimes \mathbf{x}$ for $\mathbf{x} \in \mathbb{R}^{3}$. As in the twodimensional case, we re-parameterize $\partial B\left(R \mathbf{e}_{1}, R\right)$ and compute the first integral above to obtain a diagonal matrix times $\epsilon(\xi, \eta)$. Therefore, analagous to the two-dimensional case, we should choose the ansatz for $\epsilon(\xi, \eta)$ in such a way so that

$$
\int_{-\pi}^{\pi} \int_{0}^{\pi} M_{2}^{i j}\left(\xi, \xi^{\prime}, \eta, \eta^{\prime}\right) \epsilon_{j}\left(\xi^{\prime}, \eta^{\prime}\right) \sin \eta^{\prime} \mathrm{d} \eta^{\prime} \mathrm{d} \xi^{\prime} \propto \epsilon_{i}(\xi, \eta) .
$$

Let us now turn to this task. To simplify the notation, let $\mathbf{x}:=\mathbf{X}(\xi, \eta)$ and $\mathbf{w}:=\mathbf{X}\left(\xi^{\prime}, \eta^{\prime}\right)$ with $\mathbf{X}(\xi, \eta)$ denoting our parameterization of the sphere. Consider the quantity $\mathbf{x} \cdot \mathbf{w}:=\mathbf{X}(\xi, \eta) \cdot \mathbf{X}\left(\xi^{\prime}, \eta^{\prime}\right)$. As $\mathbf{v}=(I-M) R \mathbf{e}_{1}$ and $\underline{\underline{\mathbf{v}}}=\left(I-M^{t}\right) R \mathbf{e}_{1}$, straightforward calculations yield

$$
\begin{aligned}
& \mathbf{v}=R\left(1-\mathbf{x} \cdot \mathbf{w},-(\mathbf{x} \cdot \mathbf{w})_{\eta},-\frac{(\mathbf{x} \cdot \mathbf{w})_{\xi}}{\sin (\eta)}\right)^{t}|\mathbf{v}|^{2}=2 R^{2}(1-\mathbf{x} \cdot \mathbf{w}) \\
& \underline{\underline{\mathbf{v}}}=R\left(1-\mathbf{x} \cdot \mathbf{w},-(\mathbf{x} \cdot \mathbf{w})_{\eta^{\prime}},-\frac{(\mathbf{x} \cdot \mathbf{w})_{\xi^{\prime}}}{\sin \left(\eta^{\prime}\right)}\right)^{t} .
\end{aligned}
$$

We now make the key observation that $M_{2}^{11}$ depends only upon the quantity $\mathbf{x} \cdot \mathbf{w}$, in that $M_{2}^{11}\left(\xi, \xi^{\prime}, \eta, \eta^{\prime}\right)=$ $g_{1}(\mathbf{x} \cdot \mathbf{w})$ for $g_{1}(s)=R^{2} g_{s}\left(R^{2}(1-s)\right)(1-s)^{2}-g\left(R^{2}(1-s)\right) s$. For such functions, we shall make repeated use the following (c.f. [32):

Theorem 3.1. (Funk-Hecke Theorem in 3D) Let $f(s) \in L^{1}([-1,1])$. Then for any spherical harmonic $S^{l}(\mathbf{x})$ of degree $l$ and $\mathbf{x} \in \mathcal{S}^{2}$,

$$
\lambda S^{l}(\mathbf{x})=\int_{\mathcal{S}^{2}} f(\mathbf{x} \cdot \mathbf{w}) S^{l}(\mathbf{w}) \mathrm{d} \mathcal{S}_{\mathbf{w}} .
$$

The eigenvalue $\lambda$ depends only on the function $f$ and the degree $l$ of the harmonic, where we will now write $\lambda=\lambda_{l}(f)$ to make explicit the dependencies of the eigenvalues on the functions involved. More specifically,

$$
\lambda_{l}(f)=2 \pi \int_{-1}^{1} f(s) P_{l}(s) \mathrm{d} s,
$$

where $P_{l}(s)$ denotes the Legendre polynomial of degree $l$, normalized to $P_{l}(1)=1$. In three dimensions, equation (23) plays the role that equation (20) serves in two dimensions.

Together with our observation regarding $M_{2}^{11}$, this theorem suggests we choose ansatz with $\epsilon_{1}(\xi, \eta)=$ $c_{1} S^{l}(\mathbf{x}(\xi, \eta))$ for some coefficient $c_{1} \in \mathbb{R}$. Indeed, then

$$
\int_{-\pi}^{\pi} \int_{0}^{\pi} M_{2}^{11}\left(\xi, \xi^{\prime}, \eta, \eta^{\prime}\right) \epsilon_{1}\left(\xi^{\prime}, \eta^{\prime}\right) \sin \eta^{\prime} \mathrm{d} \eta^{\prime} \mathrm{d} \xi^{\prime}=c_{1} \int_{\mathcal{S}^{2}} g_{1}(\mathbf{x} \cdot \mathbf{w}) S^{l}(\mathbf{w}) \mathrm{d} \mathcal{S}_{\mathbf{w}}
$$

so that by a straightforward application of the Funk-Hecke (F-H) theorem,

$$
\int_{-\pi}^{\pi} \int_{0}^{\pi} M_{2}^{11}\left(\xi, \xi^{\prime}, \eta, \eta^{\prime}\right) \epsilon_{1}\left(\xi^{\prime}, \eta^{\prime}\right) \sin \eta^{\prime} \mathrm{d} \eta^{\prime} \mathrm{d} \xi^{\prime}=\lambda_{l}\left(g_{1}\right) c_{1} S^{l}(\mathbf{x}) \propto \epsilon_{1}
$$

as desired. With $\epsilon_{1}$ now in hand, we can use the requirement that

$$
\int_{-\pi}^{\pi} \int_{0}^{\pi} M_{2}^{i 1}\left(\xi, \xi^{\prime}, \eta, \eta^{\prime}\right) \epsilon_{1}\left(\xi^{\prime}, \eta^{\prime}\right) \sin \eta^{\prime} \mathrm{d} \eta^{\prime} \mathrm{d} \xi^{\prime} \propto \epsilon_{i}(\xi, \eta)
$$

to determine the remainder of the ansatz. For this, we observe that the relations 21$]$ and 222 imply

$$
M_{2}^{21}=\left(g\left(\frac{1}{2}|\mathbf{v}|^{2}\right)(1-\mathbf{x} \cdot \mathbf{w})\right)_{\eta} M_{2}^{31}=\frac{1}{\sin (\eta)}\left(g\left(\frac{1}{2}|\mathbf{v}|^{2}\right)(1-\mathbf{x} \cdot \mathbf{w})\right)_{\xi} .
$$


Using this with the known choice of $\epsilon_{1}$, we find the relation 25 for $i=2$ becomes

$$
\epsilon_{2}(\xi, \eta) \propto \int_{\mathcal{S}^{2}}\left(g\left(\frac{1}{2}|\mathbf{v}|^{2}\right)(1-\mathbf{x} \cdot \mathbf{w})\right)_{\eta} c_{1} S^{l}(\mathbf{w}) \mathrm{d} \mathcal{S}_{\mathbf{w}}=c_{1}\left(\int_{\mathcal{S}^{2}} g_{2}(\mathbf{x} \cdot \mathbf{w}) S^{l} \mathrm{~d} \mathcal{S}_{\mathbf{w}}\right)_{\eta}
$$

where $g_{2}(s)=g\left(R^{2}(1-s)\right)(1-s)$, by passing the derivative through the integral in the primed variables. Again using the F-H theorem, we recover $\epsilon_{2}(\xi, \eta) \propto c_{1} \lambda_{l}\left(g_{2}\right) S_{\eta}^{l}$. Arguing similarly from 25 with $i=3$ yields $\epsilon_{3}(\xi, \eta) \propto \frac{c_{1} \lambda_{l}\left(g_{2}\right)}{\sin (\eta)} S_{\xi}^{l}$. All together, we recover

$$
\epsilon(\xi, \eta)=\left(c_{1} S^{l}(\mathbf{x}), c_{2}\left(S^{l}(\mathbf{x})\right)_{\eta}, c_{3} \frac{\left(S^{l}(\mathbf{x})\right)_{\xi}}{\sin (\eta)}\right)^{t}
$$

for real coefficients $c_{i}$.

Proceeding as in the two-dimensional case, it remains to show that this choice of $\epsilon(\xi, \eta)$ yields

$$
\int_{-\pi}^{\pi} \int_{0}^{\pi} M_{2}(\cdot) \epsilon\left(\xi^{\prime}, \eta^{\prime}\right) \sin \eta^{\prime} \mathrm{d} \eta^{\prime} \mathrm{d} \xi^{\prime}=D\left(c_{1}, c_{2}, c_{3}, l\right)\left(S^{l}, S_{\eta}^{l}, \frac{S_{\xi}^{l}}{\sin (\eta)}\right)^{t},
$$

where $D\left(c_{1}, c_{2}, c_{3}, l\right)$ is a constant, diagonal matrix that is linear in the coefficients. The derivation of the ansatz has demonstrated this claim for the first column of $M_{2}$. Continuing with the remainder first row of $M_{2}$, in light of $(21)$ and 22 we see

$$
M_{2}^{12}=\left(g\left(\frac{1}{2}|\mathbf{v}|^{2}\right)(1-\mathbf{x} \cdot \mathbf{w})\right)_{\eta^{\prime}} M_{2}^{13}=\frac{1}{\sin \left(\eta^{\prime}\right)}\left(g\left(\frac{1}{2}|\mathbf{v}|^{2}\right)(1-\mathbf{x} \cdot \mathbf{w})\right)_{\xi^{\prime}} .
$$

In setting $c_{2}=c_{3}$, we compute $\int_{-\pi}^{\pi} \int_{0}^{\pi}\left(M_{2}^{12} \epsilon_{2}+M_{2}^{13} \epsilon_{3}\right) \sin \eta^{\prime} \mathrm{d} \eta^{\prime} \mathrm{d} \xi^{\prime}=$

$$
c_{2} \int_{-\pi}^{\pi} \int_{0}^{\pi}\left(g_{2}(\mathbf{x} \cdot \mathbf{w})\right)_{\eta^{\prime}} S^{l}(\mathbf{w})_{\eta^{\prime}} \sin \eta^{\prime} \mathrm{d} \eta^{\prime} \mathrm{d} \xi^{\prime}+c_{2} \int_{-\pi}^{\pi} \int_{0}^{\pi} \frac{\left(g_{2}(\mathbf{x} \cdot \mathbf{w})\right)_{\xi^{\prime}} S_{\xi^{\prime}}^{l}}{\sin \left(\eta^{\prime}\right)^{2}} \sin \eta^{\prime} \mathrm{d} \eta^{\prime} \mathrm{d} \xi^{\prime} .
$$

Integrating by parts in $\eta^{\prime}$ in the first term and in $\xi^{\prime}$ in the second term, we have

$$
\int_{-\pi}^{\pi} \int_{0}^{\pi}\left(M_{2}^{12} \epsilon_{2}+M_{2}^{13} \epsilon_{3}\right) \sin \eta^{\prime} \mathrm{d} \eta^{\prime} \mathrm{d} \xi^{\prime}=-c_{2} \int_{-\pi}^{\pi} \int_{0}^{\pi}\left(\Delta_{\mathcal{S}^{2}} S^{l}\right) g_{2}(\mathbf{x} \cdot \mathbf{w}) \sin \eta^{\prime} \mathrm{d} \eta^{\prime} \mathrm{d} \xi^{\prime} .
$$

Using that $\Delta_{\mathcal{S}^{2}} S^{l}=-l(l+1) S^{l}$ and the F-H theorem, we obtain $\int_{-\pi}^{\pi} \int_{0}^{\pi}\left(M_{2}^{12} \epsilon_{2}+M_{2}^{13} \epsilon_{3}\right) \sin \eta^{\prime} \mathrm{d} \eta^{\prime} \mathrm{d} \xi^{\prime}=$ $c_{2} l(l+1) \lambda_{l}\left(g_{2}\right) S^{l}(\mathbf{x}) \propto \epsilon_{1}$ as desired. Proceeding similarly with the remainder of the second row of $M_{2}$, the facts

$$
\begin{gathered}
M_{2}^{22}=-\left(g\left(\frac{1}{2}|\mathbf{v}|^{2}\right)\right)_{\eta^{\prime}}(\mathbf{x} \cdot \mathbf{w})_{\eta}-g\left(\frac{1}{2}|\mathbf{v}|^{2}\right)(\mathbf{x} \cdot \mathbf{w})_{\eta \eta^{\prime}}, \\
M_{2}^{23}=\frac{-\left(g\left(\frac{1}{2}|\mathbf{v}|^{2}\right)\right)_{\xi^{\prime}}(\mathbf{x} \cdot \mathbf{w})_{\eta}-g\left(\frac{1}{2}|\mathbf{v}|^{2}\right)(\mathbf{x} \cdot \mathbf{w})_{\eta \xi^{\prime}}}{\sin \left(\eta^{\prime}\right)}
\end{gathered}
$$

and a similar integration by parts combine to give

$$
\begin{gathered}
\int_{-\pi}^{\pi} \int_{0}^{\pi}\left(M_{2}^{22} \epsilon_{2}+M_{2}^{23} \epsilon_{3}\right) \sin \eta^{\prime} \mathrm{d} \eta^{\prime} \mathrm{d} \xi^{\prime}=c_{2} \int_{-\pi}^{\pi} \int_{0}^{\pi} g\left(\frac{1}{2}|\mathbf{v}|^{2}\right)(\mathbf{x} \cdot \mathbf{w})_{\eta}\left(\Delta_{\mathcal{S}^{2}} S^{l}\right) \mathrm{d} \mathcal{S}_{2} \\
=-c_{2} l(l+1) \int_{-\pi}^{\pi} \int_{0}^{\pi} g\left(\frac{1}{2}|\mathbf{v}|^{2}\right)(\mathbf{x} \cdot \mathbf{w})_{\eta} S^{l}(\mathbf{w}) \sin \eta^{\prime} \mathrm{d} \eta^{\prime} \mathrm{d} \xi^{\prime}
\end{gathered}
$$

Letting $g_{3}(s)=\int_{0}^{R^{2}(1-s)} g(z) \mathrm{d} z$, so that $g_{3}(\mathbf{x} \cdot \mathbf{w})_{\eta}=-R^{2} g\left(\frac{1}{2}|\mathbf{v}|^{2}\right)(\mathbf{x} \cdot \mathbf{w})_{\eta}$, we pass the derivative through the integral and use the F-H theorem as before to arrive at

$$
\int_{-\pi}^{\pi} \int_{0}^{\pi}\left(M_{2}^{22} \epsilon_{2}+M_{2}^{23} \epsilon_{3}\right) \sin \eta^{\prime} \mathrm{d} \eta^{\prime} \mathrm{d} \xi^{\prime}=\frac{c_{2} l(l+1) \lambda_{l}\left(g_{3}\right)}{R^{2}} S_{\eta}^{l} \propto \epsilon_{2}
$$


Finally, the same argument on the remainder of the last row of $M_{2}$ gives

$$
\int_{-\pi}^{\pi} \int_{0}^{\pi}\left(M_{2}^{32} \epsilon_{2}+M_{2}^{33} \epsilon_{3}\right) \sin \eta^{\prime} \mathrm{d} \eta^{\prime} \mathrm{d} \xi^{\prime}=\frac{c_{2} l(l+1) \lambda_{l}\left(g_{3}\right)}{R^{2} \sin (\eta)} S_{\xi}^{l} \propto \epsilon_{3}
$$

Combining all of the above, we find

$$
\begin{gathered}
\int_{-\pi}^{\pi} \int_{0}^{\pi} M_{2}\left(\xi, \xi^{\prime}, \eta, \eta^{\prime}\right) \epsilon\left(\xi^{\prime}, \eta^{\prime}\right) \sin \eta^{\prime} \mathrm{d} \eta^{\prime} \mathrm{d} \xi^{\prime}=\left(D^{11} S^{l}, D^{22} S_{\eta}^{l}, D^{33} \frac{S_{\xi}^{l}}{\sin (\eta)}\right)^{t}, \\
D^{11}=c_{1} \lambda_{l}\left(g_{1}\right)+c_{2} l(l+1) \lambda_{l}\left(g_{2}\right) D^{22}=D^{33}=c_{1} \lambda_{l}\left(g_{2}\right)+c_{2} \frac{l(l+1)}{R^{2}} \lambda_{l}\left(g_{3}\right),
\end{gathered}
$$

so that $D$ is linear in the coefficients as desired. Consequently, with this ansatz the linearized equations (17) become

$$
\lambda \epsilon(\xi, \eta)=\left(\int_{\partial B\left(R \mathbf{e}_{1}, R\right)} G(\mathbf{x}) \mathrm{d} S(\mathbf{x})\right) \epsilon(\xi, \eta)+D\left(c_{1}, c_{2}, l\right) \epsilon(\xi, \eta) .
$$

By symmetry, we see

$$
\left(\int_{\partial B\left(R \mathbf{e}_{1}, R\right)} G(\mathbf{x}) \mathrm{d} S(\mathbf{x})\right)=\operatorname{diag}(\alpha, \beta, \beta),
$$

so that the second and third equations in 27 are identical. Consequently, solving the continuous linearized equations reduces to the $2 \times 2$ scalar eigenvalue problem determined by 27$]: \lambda c_{1}=\alpha c_{1}+D^{11}\left(c_{1}, c_{2}, l\right)$ and $\lambda c_{2}=\beta c_{2}+D^{22}\left(c_{1}, c_{2}, l\right)$, just as in the two-dimensional case.

We can make one final simplification to the eigenvalue problem that comes from the steady-state equation for the sphere,

$$
0=\int_{\mathcal{S}^{2}} g\left(\frac{R^{2}}{2}|\mathbf{x}-\mathbf{w}|^{2}\right)(\mathbf{x}-\mathbf{w}) \mathrm{d} \mathcal{S}_{\mathbf{w}} .
$$

In particular, the sphere radius $R$ satisfies (see Remark 3.2 below)

$$
0=\int_{\mathcal{S}^{2}} g\left(R^{2}(1-\mathbf{x} \cdot \mathbf{w})\right)(1-\mathbf{x} \cdot \mathbf{w}) \mathrm{d} S_{y} \Leftrightarrow 0=\int_{-1}^{1} g_{2}(s) \mathrm{d} s
$$

by the $\mathrm{F}-\mathrm{H}$ theorem with $l=0$. A simple calculation then gives $\beta=0$.

To summarize the previous calculations, the decoupled sequence of eigenvalue problems

$$
\lambda\left(\begin{array}{l}
c_{1} \\
c_{2}
\end{array}\right)=\left(\begin{array}{cc}
\alpha+\lambda_{l}\left(g_{1}\right) & l(l+1) \lambda_{l}\left(g_{2}\right) \\
\lambda_{l}\left(g_{2}\right) & \frac{l(l+1)}{R^{2}} \lambda_{l}\left(g_{3}\right)
\end{array}\right)\left(\begin{array}{l}
c_{1} \\
c_{2}
\end{array}\right):=\Omega_{l}\left(\begin{array}{c}
c_{1} \\
c_{2}
\end{array}\right)
$$

determine the linear stability of the uniform sphere. To compute the entries of $\Omega_{l}$, we recall that for a function $h \in L^{1}([-1,1])$ and $l \in \mathbb{N}$ we define $\lambda_{l}(h)=2 \pi \int_{-1}^{1} h(s) P_{l}(s) \mathrm{d} s$, with $P_{l}(s)$ denoting the Legendre polynomial of degree $l$ normalized to $P_{l}(1)=1$. The radius $R$ of the sphere satisfies $0=\int_{-1}^{1} g\left(R^{2}(1-s)\right)(1-s) \mathrm{d} s$ and the coefficient $\alpha=8 \pi g\left(2 R^{2}\right)+2 \pi \int_{-1}^{1} g\left(R^{2}(1-s)\right) \mathrm{d} s$. Finally, we recall the definitions

$g_{1}(s)=R^{2} g_{s}\left(R^{2}(1-s)\right)(1-s)^{2}-g\left(R^{2}(1-s)\right) s, \quad g_{2}(s)=g\left(R^{2}(1-s)\right)(1-s), \quad\left(g_{3}\right)_{s}(s)=-R^{2} g\left(R^{2}(1-s)\right)$.

Remark 3.2. For sufficiently smooth attractive-repulsive interaction kernels g, a uniform density, steadystate sphere solution to equation (13) exists if and only if $-\infty \leq \int_{0}^{\infty} s g(s) \mathrm{d} s<0$. Indeed, projecting the equation of steady-state 28 onto the component normal to the sphere yields

$$
0=\int_{\mathcal{S}^{2}} g\left(R^{2}(1-\mathbf{x} \cdot \mathbf{w})\right)(1-\mathbf{x} \cdot \mathbf{w}) \mathrm{d} S_{y} \Leftrightarrow 0=\int_{-1}^{1} g_{2}(s) \mathrm{d} s
$$


by the $F-H$ theorem with $l=0$. Since $g>0$ near zero, for $R$ sufficiently small the integral on the RHS of (32) is positive. Similarly, since $g<0$ away from the origin, for $R$ large enough the integral on the RHS decreases as $R \rightarrow \infty$. Thus when $\int_{0}^{\infty} s g(s) \mathrm{d} s<0$ the $R H S$ of 32 is negative for all sufficiently large radii. Therefore there exists an $R$ which identically satisfies $(32)$. That the projection onto the tangential components satisfies (28) follows in a manner similar to the derivation of the tangential components of the ansatz. This applies regardless of the stability of the sphere, and regardless of whether the potential exhibits confinement.

Remark 3.3. In concordence with a local notion like linear stability, all equations only involve values of the potential $V$ and its derivatives for values of $s \in\left[0,2 R^{2}\right]$. In particular, once we know the radius $R$ of the sphere, we can assign arbitrary far-field behavior to the potential without affecting the validity or applicability of our analysis.

\subsection{Linearization of the Density}

By solving the eigenvalue problem (30), our work in the previous section allows us to construct approximate solutions to the surface equations (12) with $f_{0}(\xi, \eta)=\sin (\eta)$, which correspond to small perturbations of the spherical solution. As we mentioned in $\S 2$, knowledge of the surface allows us to reconstruct the density of particles via $\rho_{S}(\xi, \eta, t)\left|\mathbf{X}_{\xi} \times \mathbf{X}_{\eta}\right|(t)=f_{0}(\xi, \eta)$. Therefore, a perturbation away from a sphere naturally induces a perturbation of the density away from uniform as well. Indeed, if we write our perturbation of the sphere as $u(\mathbf{x}, t)=\Theta_{1}(\xi) \Theta_{2}(\eta)\left(R \mathbf{e}_{1}+\epsilon(\mathbf{x}) e^{\lambda t}\right)$ for $\mathbf{x}(\xi, \eta) \in \mathcal{S}^{2}$ and $\epsilon(\mathbf{x})$ as in equation (26), we can linearize $\rho_{S}=\frac{\sin (\eta)}{\left|u_{\xi} \times u_{\eta}\right|}$ to find the leading order corrections to the density.

To this end, define $\mathcal{B}(\xi, \eta):=\Theta_{1}(\xi) \Theta_{2}(\eta)$. We can then compute $\left|u_{\xi} \times u_{\eta}\right|$ by using Lagrange's identity:

$$
\left|u_{\xi} \times u_{\eta}\right|^{2}=\left(u_{\xi} \cdot u_{\xi}\right)\left(u_{\eta} \cdot u_{\eta}\right)-\left(u_{\xi} \cdot u_{\eta}\right)^{2} .
$$

Computing this directly, we find

$$
\begin{gathered}
\left|u_{\xi}\right|^{2}=\left|\mathcal{B}_{\xi} R \mathbf{e}_{1}\right|^{2}+2 R \mathcal{B}_{\xi} \mathbf{e}_{1} \cdot\left(\mathcal{B}_{\xi} \epsilon(\mathbf{x})+\mathcal{B} \epsilon(\mathbf{x})_{\xi}\right) e^{\lambda t}+\mathcal{O}\left(\epsilon^{2}\right) \\
\left|u_{\eta}\right|^{2}=\left|\mathcal{B}_{\eta} R \mathbf{e}_{1}\right|^{2}+2 R \mathcal{B}_{\eta} \mathbf{e}_{1} \cdot\left(\mathcal{B}_{\eta} \epsilon(\mathbf{x})+\mathcal{B} \epsilon(\mathbf{x})_{\eta}\right) e^{\lambda t}+\mathcal{O}\left(\epsilon^{2}\right) \\
u_{\xi} \cdot u_{\eta}=R^{2}\left(\mathcal{B}_{\eta}^{t} \mathcal{B}_{\xi}\right)^{11}+\mathcal{O}(\epsilon)
\end{gathered}
$$

Straightforward calculations yield the required derivatives of $\mathcal{B}$ :

$$
\begin{gathered}
\mathcal{B}_{\xi}^{t} \mathcal{B}_{\xi}=\left(\begin{array}{ccc}
\sin ^{2}(\eta) & \sin (\eta) \cos (\eta) & 0 \\
\sin (\eta) \cos (\eta) & \cos ^{2}(\eta) & 0 \\
0 & 0 & 1
\end{array}\right) \quad \mathcal{B}^{t} \mathcal{B}_{\xi}=\left(\begin{array}{ccc}
0 & 0 & -\sin (\eta) \\
0 & 0 & -\cos (\eta) \\
\sin (\eta) & \cos (\eta) & 0
\end{array}\right) \\
\mathcal{B}_{\eta}^{t} \mathcal{B}_{\eta}=\left(\begin{array}{lll}
1 & 0 & 0 \\
0 & 1 & 0 \\
0 & 0 & 0
\end{array}\right) \\
\mathcal{B}^{t} \mathcal{B}_{\eta}=\left(\begin{array}{ccc}
0 & -1 & 0 \\
1 & 0 & 0 \\
0 & 0 & 0
\end{array}\right) \quad \mathcal{B}_{\eta}^{t} \mathcal{B}_{\xi}=\left(\begin{array}{ccc}
0 & 0 & -\cos (\eta) \\
0 & 0 & \sin (\eta) \\
0 & 0 & 0
\end{array}\right) .
\end{gathered}
$$

Using the relations (33) and (34) and the definition of the ansatz (26), we obtain

$$
\begin{gathered}
\left|u_{\xi}\right|^{2}=\sin ^{2}(\eta)\left\{R^{2}+2 R c_{1} e^{\lambda t} S^{l}+2 R c_{2} e^{\lambda t}\left(\cot (\eta) S_{\eta}^{l}+\frac{S_{\xi \xi}^{l}}{\sin ^{2}(\eta)}\right)\right\}+\mathcal{O}\left(\epsilon^{2}\right) \\
\left|u_{\eta}\right|^{2}=R^{2}+2 R e^{\lambda t}\left\{c_{1} S^{l}+c_{2} S_{\eta \eta}^{l}\right\}+\mathcal{O}\left(\epsilon^{2}\right),\left(u_{\xi} \cdot u_{\eta}\right)^{2}=\mathcal{O}\left(\epsilon^{2}\right) .
\end{gathered}
$$

Therefore, $\left|u_{\xi} \times u_{\eta}\right|^{2}=\sin ^{2}(\eta)\left\{R^{4}+4 c_{1} e^{\lambda t} R^{3} S^{l}+2 c_{2} e^{\lambda t} R^{3}\left(\Delta_{\mathcal{S}^{2}} S^{l}\right)\right\}+\mathcal{O}\left(\epsilon^{2}\right)$, so that to leading order the perturbed density $\rho_{S}$ obeys

$$
\rho_{S}=\frac{1}{R^{2}}\left\{1-\frac{e^{\lambda t}}{R}\left(2 c_{1}-c_{2} l(l+1)\right) S^{l}(\mathbf{x})\right\}+\mathcal{O}\left(\epsilon^{2}\right)
$$


Additionally, we can determine the principal correction to the radius of the surface,

$$
|u(\mathbf{x}, t)|=\sqrt{R^{2}+2 c_{1} e^{\lambda t} R S^{l}(\mathbf{x})+\mathcal{O}\left(\epsilon^{2}\right)}=R\left(1+\frac{c_{1} e^{\lambda t}}{R} S^{l}(\mathbf{x})\right)+\mathcal{O}\left(\epsilon^{2}\right) .
$$

We may therefore view the modes determined by the eigenvalue problems $(30)$ as spheres of variable radius $R+c_{1} e^{\lambda t} S^{l}(\mathbf{x})$, with the non-uniform particle density determined from $(35)$.

\section{Eigenvalue Problem in Arbitrary Dimensions}

In this section, we extend our analysis of the linearization of the surface equation and the reduction to a scalar eigenvalue problem to an arbitrary $(d-1)$-sphere. Although the notation is more cumbersome, the argument proceeds exactly as in the three-dimensional case. Additionally, due to the tangential isotropy of the sphere, the matrix associated to the eigenvalue problem remains $2 \times 2$, regardless of dimension.

In the $d$-dimensional setting, the analogue of 13 becomes

$$
\begin{aligned}
& \mathbf{X}_{t}\left(\eta_{1}, \ldots, \eta_{d-1}, t\right)=\int_{\mathcal{S}^{d-1}} g\left(\frac{1}{2}\left|\mathbf{X}-\mathbf{X}^{\prime}\right|^{2}\right)\left(\mathbf{X}-\mathbf{X}^{\prime}\right) \mathrm{d} \mathcal{S}_{d-1} \\
& \mathbf{X}=\mathbf{X}\left(\eta_{1}, \ldots, \eta_{d-1}, t\right) \in \mathbb{R}^{d} \mathbf{X}^{\prime}=\mathbf{X}\left(\eta_{1}^{\prime}, \ldots, \eta_{d-1}^{\prime}, t\right) \in \mathbb{R}^{d},
\end{aligned}
$$

so that a uniformly distributed steady-state sphere of radius $R$ satisfies

$$
0=\int_{\mathcal{S}^{d-1}} g\left(\frac{R^{2}}{2}|\mathbf{x}-\mathbf{w}|^{2}\right)(\mathbf{x}-\mathbf{w}) \mathrm{d} \mathcal{S}_{\mathbf{w}} \forall \mathbf{x} \in \mathcal{S}^{d-1}
$$

We write the sphere of radius $R$ in dimension $d$ as $R \mathbf{x}$ for $\mathbf{x} \in \mathcal{S}^{d-1}$, and write a perturbation in the form $\delta \mathbf{x}=R \mathbf{x}+\mathcal{B}(\mathbf{x}) \epsilon(\mathbf{x}) e^{\lambda t}$. The matrix $\mathcal{B}$ plays the role of $\Theta_{1}(\xi) \Theta_{2}(\eta)$ from the three dimensional calculation, i.e. a product of rotation matrices. We define the rows $\mathbf{b}_{j}$ of $\mathcal{B}(\mathbf{x})$ as follows: we take $\mathbf{b}_{1}=\mathbf{x}$; next, define $\hat{\mathbf{b}}_{j}(\mathbf{x})=\partial_{\eta_{j}}(\mathbf{x})$, where $\eta_{j}$ denote any of the coordinates on $\mathcal{S}^{d-1}$; lastly, normalize $\mathbf{b}_{j}(\mathbf{x})=\frac{\hat{\mathbf{b}}_{j}(\mathbf{x})}{\left|\hat{\mathbf{b}}_{j}(\mathbf{x})\right|}$. Then $\mathcal{B}^{t} \mathcal{B}(\mathbf{x})=I$ for all $\mathbf{x} \in \mathcal{S}^{d-1}$. As for the ansatz, put $\epsilon_{1}(\mathbf{x})=c_{1} S^{l}(\mathbf{x})$ as before, and $\epsilon_{j+1}(\mathbf{x})=$ $c_{2} \partial_{\eta_{j}}\left(S^{l}(\mathbf{x})\right) /\left|\hat{\mathbf{b}}_{j}(\mathbf{x})\right|$ for $1 \leq j \leq d-1$.

If we now expand to first order in $\epsilon$ and use the fact that the sphere is a steady-state, we obtain the continuous eigenvalue problem

$$
\begin{aligned}
\lambda \epsilon(\mathbf{x})= & \int_{\mathcal{S}^{d-1}}\left\{g\left(\frac{1}{2}|\mathbf{v}|^{2}\right) I+g_{s}\left(\frac{1}{2}|\mathbf{v}|^{2}\right) \mathbf{v} \otimes \mathbf{v}\right\} \epsilon(\mathbf{x}) \mathrm{d} \mathcal{S}_{\mathbf{w}}+ \\
& \int_{\mathcal{S}^{d-1}}\left\{g_{s}\left(\frac{1}{2}|\mathbf{v}|^{2}\right) \mathbf{v} \otimes \underline{\underline{\mathbf{v}}}-g\left(\frac{1}{2}|\mathbf{v}|^{2}\right) \mathcal{B}^{t}(\mathbf{x}) \mathcal{B}(\mathbf{w})\right\} \epsilon(\mathbf{w}) \mathrm{d} \mathcal{S}_{\mathbf{w}},
\end{aligned}
$$

where $\mathbf{v}=R\left(\mathbf{e}_{1}-\mathcal{B}^{t}(\mathbf{x}) \mathbf{w}\right)$ and $\underline{\underline{\mathbf{v}}}=R\left(\mathbf{e}_{1}-\mathcal{B}^{t}(\mathbf{w}) \mathbf{x}\right)$. This generalizes 17$)$ to any dimension. As in three dimensions, the reduction to a scalar eigenvalue problem follows from two claims. If we let $M_{1}$ denote the matrix in the first term of (38), we first claim that $M_{1}$ is diagonal of the form $M_{1}=\operatorname{diag}(\alpha, 0, \cdots, 0)$ and independent of $\mathbf{x}$. Second, we claim that

$$
\int_{\mathcal{S}^{d-1}} M_{2}(\mathbf{x}, \mathbf{w}) \epsilon(\mathbf{w}) \mathrm{d} \mathcal{S}_{\mathbf{w}}=\left(D^{11} S^{l}, D^{22} \frac{S_{\eta_{1}}^{l}}{\left|\hat{\mathbf{b}}_{1}\right|}, \ldots, D^{22} \frac{S_{\eta_{d-1}}^{l}}{\left|\hat{\mathbf{b}}_{d-1}\right|}\right),
$$

where $M_{2}$ denotes the matrix in the second term of $\left.\sqrt{38}\right)$, and $D^{i i}=D^{i i}\left(c_{1}, c_{2}, l\right)$ are linear in the coefficients. Combining this with the first claim again reduces 38 to the scalar eigenvalue problem

$$
\lambda\left(\begin{array}{c}
c_{1} \\
c_{2}
\end{array}\right)=\left(\begin{array}{c}
\alpha c_{1}+D^{11}\left(c_{1}, c_{2}, l\right) \\
D^{22}\left(c_{1}, c_{2}, l\right)
\end{array}\right) .
$$

To establish the claims, we once again have as our main tool ([32]): 
Theorem 4.1. (Funk-Hecke Theorem in d-dimensions) Let $f(s)\left(1-s^{2}\right)^{\frac{d-3}{2}} \in L^{1}([-1,1])$. Then for any spherical harmonic of degree $l$ and $\mathbf{x} \in \mathcal{S}^{d-1}$,

$$
\lambda S^{l}(\mathbf{x})=\int_{\mathcal{S}^{d-1}} f(\mathbf{x} \cdot \mathbf{w}) S^{l}(\mathbf{w}) \mathrm{d} \mathcal{S}_{\mathbf{w}} .
$$

Again, the eigenvalue $\lambda=\lambda_{l}(f)$ depends only on the function $f$ and the degree $l$ of the harmonic, in that

$$
\lambda_{l}(f)=\operatorname{vol}\left(\mathcal{S}^{d-2}\right) \int_{-1}^{1} f(s) P_{l, d}(s)\left(1-s^{2}\right)^{\frac{d-3}{2}} \mathrm{~d} s,
$$

where $\operatorname{vol}\left(\mathcal{S}^{d-2}\right)$ denotes the surface area of the $d-2$ sphere. Also, $P_{l, d}(s)$ denotes the Gegenbauer polynomial $P_{l}^{(d / 2-1)}(s)$ from [35], normalized so that $P_{l}(1)=1$. For $d=3$, these coincide the Legendre polynomials, whereas for $d=2$ we have $P_{l}(\cos (\eta))=\cos (l \eta)$. Under this change of variable, for $d=2$, we recover precisely the eigenvalue problem from [19. We shall also need the following elementary lemma. For the proof, we denote by $\mathbf{w}\left(\eta_{1}, \ldots, \eta_{d-1}\right)$ a parameterization of $\mathcal{S}^{d-1}$ such that $\mathbf{w}=\left(\cos \eta_{1}, \sin \eta_{1} \tilde{\mathbf{w}}\right)$ and $\tilde{\mathbf{w}}\left(\eta_{2}, \ldots, \eta_{d-1}\right)$ parametrizes $\mathcal{S}^{d-2}$. We also use Einstein notation for terms involving partial derivatives.

Lemma 1. Let $f\left(\eta_{1}, \ldots, \eta_{d-1}\right) \in C^{2}$ with $\left(\eta_{1}, \ldots, \eta_{d-1}\right)$ denoting coordinates on $\mathcal{S}^{d-1}$ as above. Then $\partial_{\eta_{j}}\left(f_{\eta_{j}} \frac{\mathrm{d} \mathcal{S}_{\mathbf{w}}}{\left|\mathbf{w}_{\eta_{j}}\right|^{2}}\right)=\Delta_{\mathcal{S}^{d-1}}(f) \mathrm{d} \mathcal{S}_{\mathbf{w}}$.

Proof. We induct on the dimension $d$. When $d=2$ this reads

$$
\partial_{\eta} f_{\eta} \mathrm{d} \eta=\Delta_{\mathcal{S}^{1}}(f) \mathrm{d} \eta
$$

so there is nothing to prove. Let us now write $\mathrm{d} \mathcal{S}_{\mathbf{w}}=\sin ^{d-2}\left(\eta_{1}\right) \mathrm{d} \mathcal{S}^{d-2}$, where $\mathrm{d} \mathcal{S}^{d-2}$ depends only on $\eta_{2}, \ldots, \eta_{d-1}$. As $\left|\mathbf{w}_{\eta_{1}}\right|=1$ and $\left|\mathbf{w}_{\eta_{j}}\right|=\sin \eta_{1}\left|\tilde{\mathbf{w}}_{\eta_{j}}\right|$ for $j>1$ we compute

$$
\begin{aligned}
\partial_{\eta_{j}}\left(f_{\eta_{j}} \frac{\mathrm{d} \mathcal{S}_{\mathbf{w}}}{\left|\mathbf{w}_{\eta_{j}}\right|^{2}}\right) & =\partial_{\eta_{1}}\left(f_{\eta_{1}} \sin ^{d-2} \eta_{1}\right) \mathrm{d} \mathcal{S}^{d-2}+\frac{\sin ^{d-2} \eta_{1}}{\sin ^{2} \eta_{1}} \partial_{\eta_{j}}\left(f_{\eta_{j}} \frac{\mathrm{d} \mathcal{S}^{d-2}}{\left|\tilde{\mathbf{w}}_{\eta_{j}}\right|^{2}}\right), \\
& =\partial_{\eta_{1}}\left(f_{\eta_{1}} \sin ^{d-2} \eta_{1}\right) \mathrm{d} \mathcal{S}^{d-2}+\frac{\sin ^{d-2} \Delta_{\mathcal{S}^{d-2}}(f) \mathrm{d} \mathcal{S}^{d-2}}{\sin ^{2} \eta_{1}}
\end{aligned}
$$

by the inductive hypothesis. However, $\mathrm{d} \mathcal{S}^{d-2}=\mathrm{d} \mathcal{S}_{\mathbf{w}} \sin ^{2-d} \eta_{1}$ so that we obtain

$$
\partial_{\eta_{j}}\left(f_{\eta_{j}} \frac{\mathrm{d} \mathcal{S}_{\mathbf{w}}}{\left|\mathbf{w}_{\eta_{j}}\right|^{2}}\right)=\left(\sin ^{2-d} \eta_{1} \partial_{\eta_{1}}\left(f_{\eta_{1}} \sin ^{d-2} \eta_{1}\right)+\frac{1}{\sin ^{2} \eta_{1}} \Delta_{\mathcal{S}^{d-2}}(f)\right) \mathrm{d} \mathcal{S}_{\mathbf{w}} .
$$

We recognize the expression in parentheses as $\Delta_{\mathcal{S}^{d-1}}(f)$.

Claim 1. In (38), $M_{1}=\operatorname{diag}(\alpha, 0, \cdots, 0)$ for some $\alpha \in \mathbb{R}$.

By the orthogonality of $\mathcal{B}$ we recognize the vector $\underline{\underline{\mathbf{v}}}$ as a parameterization of $\partial B\left(R \mathbf{e}_{1}, R\right)$ in $\mathbb{R}^{d}$. Thus, the first term in $(38)$ amounts to multiplying $\epsilon(\mathbf{x})$ by constant matrix

$$
M_{1}=\int_{\partial B\left(R \mathbf{e}_{1}, R\right)} g\left(\frac{1}{2}|\mathbf{x}|^{2}\right) I+g_{s}\left(\frac{1}{2}|\mathbf{x}|^{2}\right)(\mathbf{x} \otimes \mathbf{x}) \mathrm{d} \mathcal{S}_{\mathbf{x}} .
$$

Parametrize $\partial B\left(R \mathbf{e}_{1}, R\right)$ by $\mathbf{x}=R\left(\mathbf{e}_{1}-\mathbf{w}\right)$, where $\mathbf{w}=\left(\cos \left(\eta_{1}\right), \sin \left(\eta_{1}\right) \tilde{\mathbf{w}}\right)$ and $\tilde{\mathbf{w}} \in \mathbb{R}^{d-1}$ parametrizes $\mathcal{S}^{d-2}$. Then for $j, k \neq 1$ we have

$$
\begin{aligned}
M_{1}^{j k} & =\int_{0}^{\pi} \int_{\mathcal{S}^{d-2}} g\left(R^{2}\left(1-\cos \left(\eta_{1}\right)\right)\right) \delta_{j k} \sin \left(\eta_{1}\right)^{d-2} \mathrm{~d} \eta_{1} \mathrm{~d} \mathcal{S}^{d-2}+ \\
& R^{2} \int_{0}^{\pi} \int_{\mathcal{S}^{d-2}} g_{s}\left(R^{2}\left(1-\cos \left(\eta_{1}\right)\right)\right)\left(\tilde{\mathbf{w}} \cdot \mathbf{e}_{j}\right)\left(\tilde{\mathbf{w}} \cdot \mathbf{e}_{k}\right) \sin \left(\eta_{1}\right)^{d} \mathrm{~d} \eta_{1} \mathrm{~d} \mathcal{S}^{d-2} \\
& =\operatorname{vol}\left(\mathcal{S}^{d-2}\right) \delta_{j k} \int_{-1}^{1} g\left(R^{2}(1-s)\right)\left(1-s^{2}\right)^{\frac{d-3}{2}} \mathrm{~d} s \\
& +\left(\int_{\mathcal{S}^{d-2}} \mathbf{x}_{j} \mathbf{x}_{k} \mathrm{~d} \mathcal{S}^{d-2}\right) \int_{-1}^{1} R^{2} g_{s}\left(R^{2}(1-s)\right)\left(1-s^{2}\right)^{\frac{d-1}{2}} \mathrm{~d} s .
\end{aligned}
$$


By symmetry, $\int_{\mathcal{S}^{d-2}} \mathbf{x}_{j} \mathbf{x}_{k} \mathrm{~d} \mathcal{S}^{d-2}=\frac{\operatorname{vol}\left(\mathcal{S}^{d-2}\right)}{d-1} \delta_{j k}$, so that $M_{1}^{j k}=$

$$
\delta_{j k} \operatorname{vol}\left(\mathcal{S}^{d-2}\right) \int_{-1}^{1}\left(g\left(R^{2}(1-s)\right)+\frac{R^{2}}{d-1} g_{s}\left(R^{2}(1-s)\right)\left(1-s^{2}\right)\right)\left(1-s^{2}\right)^{\frac{d-3}{2}} \mathrm{~d} s
$$

whenever $j, k \neq 1$. Integrating the last term by parts, we arrive at

$$
M_{1}^{j k}=\delta_{j k} \beta:=\delta_{j k} \operatorname{vol}\left(\mathcal{S}^{d-2}\right)\left(\int_{-1}^{1} g\left(R^{2}(1-s)\right)(1-s)\left(1-s^{2}\right)^{\frac{d-3}{2}} \mathrm{~d} s\right)
$$

for $j, k>1$. Thus, the lower $(d-1) \times(d-1)$ block of $M_{1}$ takes the form $\operatorname{diag}(\beta, \ldots, \beta)$, with $\beta$ as in (39).

However, for $R \mathbf{x}$ to satisfy the equation of steady-state, we require $0=\int_{\mathcal{S}^{d-1}} g\left(R^{2}(1-\mathbf{x} \cdot \mathbf{w})\right)(1-\mathbf{x} \cdot \mathbf{w}) \mathrm{d} \mathcal{S}_{\mathbf{w}}$. Utilizing the F-H theorem with $l=0$, this gives

$$
0=\int_{-1}^{1} g\left(R^{2}(1-s)\right)(1-s)\left(1-s^{2}\right)^{\frac{d-3}{2}} \mathrm{~d} s
$$

so that in fact $\beta=0$.

We now consider the remaining entries of $M_{1}$. If we now let $j=1, k>1$ we have $M_{1}^{1 k}=M_{1}^{k 1}$

$$
\begin{aligned}
& =-R^{2} \int_{0}^{\pi} \int_{\mathcal{S}^{d-2}} g_{s}\left(R^{2}\left(1-\cos \left(\eta_{1}\right)\right)\right)\left(1-\cos \left(\eta_{1}\right)\right)\left(\tilde{\mathbf{w}} \cdot \mathbf{e}_{k}\right) \sin \left(\eta_{1}\right)^{d-1} \mathrm{~d} \eta_{1} \mathrm{~d} \mathcal{S}^{d-2} \\
& =-R^{2}\left(\int_{\mathcal{S}^{d-2}} \mathbf{x}_{k} \mathrm{~d} \mathcal{S}^{d-2}\right) \int_{-1}^{1} g_{s}\left(R^{2}(1-s)\right)(1-s)\left(1-s^{2}\right)^{\frac{d-2}{2}} \mathrm{~d} s=0 .
\end{aligned}
$$

Thus, $M_{1}=\operatorname{diag}(\alpha, 0, \ldots, 0)$ as claimed, where

$$
\alpha=\operatorname{vol}\left(\mathcal{S}^{d-2}\right) \int_{-1}^{1}\left(g\left(R^{2}(1-s)\right)+R^{2} g_{s}\left(R^{2}(1-s)\right)(1-s)^{2}\right)\left(1-s^{2}\right)^{\frac{d-3}{2}} \mathrm{~d} s .
$$

Claim 2. In 38,

$$
\int_{\mathcal{S}^{d-1}} M_{2}(\mathbf{x}, \mathbf{w}) \epsilon(\mathbf{w}) \mathrm{d} \mathcal{S}_{\mathbf{w}}=\left(D^{11} S^{l}, D^{22} \frac{S_{\eta_{1}}^{l}}{\left|\hat{\mathbf{b}}_{1}\right|}, \ldots, D^{22} \frac{S_{\eta_{d-1}}^{l}}{\left|\hat{\mathbf{b}}_{d-1}\right|}\right)
$$

where $D^{i i}=D^{i i}\left(c_{1}, c_{2}, l\right)$ are linear in $\left(c_{1}, c_{2}\right)$.

Let $\mathbf{x}=\mathbf{x}\left(\eta_{1}, \ldots, \eta_{d-1}\right)$ and $\mathbf{w}=\mathbf{w}\left(\eta_{1}^{\prime}, \ldots, \eta_{d-1}^{\prime}\right)$. We can then write the entries of $\mathbf{v}$ and $\underline{\underline{\mathbf{v}}}$ as

$$
\frac{\mathbf{v}^{1}}{R}=(1-\mathbf{x} \cdot \mathbf{w}), \frac{\mathbf{v}^{j}}{R}=-\frac{\partial_{\eta_{j}}(\mathbf{x} \cdot \mathbf{w})}{\left|\hat{\mathbf{b}}_{j}\right|(\mathbf{x})}, \frac{\underline{\underline{\mathbf{v}}}^{1}}{R}=(1-\mathbf{x} \cdot \mathbf{w}), \frac{\underline{\underline{\mathbf{v}}}^{j}}{R}=-\frac{\partial_{\eta_{j}^{\prime}}(\mathbf{x} \cdot \mathbf{w})}{\left|\hat{\mathbf{b}}_{j}\right|(\mathbf{w})},
$$

and the entries of $\mathcal{M}(\mathbf{x}, \mathbf{w}):=\mathcal{B}^{t}(\mathbf{x}) \mathcal{B}(\mathbf{w})$ as

$$
\mathcal{M}^{11}=\mathbf{x} \cdot \mathbf{w}, \quad \mathcal{M}^{1 j}=\frac{\partial_{\eta_{j}^{\prime}}(\mathbf{x} \cdot \mathbf{w})}{\left|\hat{\mathbf{b}}_{j}(\mathbf{w})\right|}, \quad \mathcal{M}^{j 1}=\frac{\partial_{\eta_{j}}(\mathbf{x} \cdot \mathbf{w})}{\left|\hat{\mathbf{b}}_{j}(\mathbf{x})\right|}, \quad \mathcal{M}^{j k}=\frac{\partial_{\eta_{j}} \partial_{\eta_{k}^{\prime}}(\mathbf{x} \cdot \mathbf{w})}{\left|\hat{\mathbf{b}}_{j}(\mathbf{x})\right|\left|\hat{\mathbf{b}}_{j}(\mathbf{w})\right|} .
$$

We now demonstrate the claim row-by-row, and in doing so, we use Einstein summation notation. Based on the preceding definitions,

$$
\begin{aligned}
& \int_{\mathcal{S}^{d-1}} M_{2}^{1 j} \epsilon_{j} \mathrm{~d} \mathcal{S}_{\mathbf{w}}=c_{1} \int_{\mathcal{S}^{d-1}} g_{1}(\mathbf{x} \cdot \mathbf{w}) S^{l}(\mathbf{w}) \mathrm{d} \mathcal{S}_{\mathbf{w}}+ \\
& c_{2} \int_{\mathcal{S}^{d-1}} \partial_{\eta_{j}^{\prime}}\left(g_{2}(\mathbf{x} \cdot \mathbf{w})\right) \partial^{\eta_{j}^{\prime}}\left(S^{l}(\mathbf{w})\right) \frac{\mathrm{d} \mathcal{S}_{\mathbf{w}}}{\left|\hat{\mathbf{b}}_{j}(\mathbf{w})\right|^{2}},
\end{aligned}
$$


where $g_{1}(s)=R^{2} g_{s}\left(R^{2}(1-s)\right)(1-s)^{2}-g\left(R^{2}(1-s)\right) s$ and $g_{2}(s)=g\left(R^{2}(1-s)\right)(1-s)$. For the first term in 42 , we use a straightforward application of the F-H theorem to obtain

$$
c_{1} \int_{\mathcal{S}^{d-1}} g_{1}(\mathbf{x} \cdot \mathbf{w}) S^{l}(\mathbf{w}) \mathrm{d} \mathcal{S}_{\mathbf{w}}=c_{1} \lambda_{l}\left(g_{1}\right) S^{l}(\mathbf{x}) .
$$

We then integrate the second term in 42 by parts to obtain

$$
\int_{\mathcal{S}^{d-1}} M_{2}^{1 j} \epsilon_{j} \mathrm{~d} \mathcal{S}_{\mathbf{w}}=c_{1} \lambda_{l}\left(g_{1}\right) S^{l}(\mathbf{x})-c_{2} \int_{\mathcal{S}^{d-1}} g_{2}(\mathbf{x} \cdot \mathbf{w}) \partial_{\eta_{j}^{\prime}}\left(\partial^{\eta_{j}^{\prime}}\left(S^{l}(\mathbf{w})\right) \frac{\mathrm{d} \mathcal{S}_{\mathbf{w}}}{\left|\hat{\mathbf{b}}_{j}(\mathbf{w})\right|^{2}}\right) .
$$

Using the lemma with $f=S^{l}(\mathbf{w}), 43$ simplifies to

$$
\begin{aligned}
\int_{\mathcal{S}^{d-1}} M_{2}^{1 j} \epsilon_{j} \mathrm{~d} \mathcal{S}_{\mathbf{w}} & =c_{1} \lambda_{l}\left(g_{1}\right) S^{l}(\mathbf{x})-c_{2} \int_{\mathcal{S}^{d-1}} g_{2}(\mathbf{x} \cdot \mathbf{w}) \Delta_{\mathcal{S}^{d-1}}\left(S^{l}(\mathbf{w})\right) \mathrm{d} \mathcal{S}_{\mathbf{w}} \\
& =\left(c_{1} \lambda_{l}\left(g_{1}\right)+c_{2} l(l+d-2) \lambda_{l}\left(g_{2}\right)\right) S^{l}(\mathbf{x}),
\end{aligned}
$$

as $S^{l}$ is an eigenfunction with eigenvalue $-l(l+d-2)$ and the F-H theorem. This establishes the claim for the first row with $D^{11}\left(c_{1}, c_{2}, l\right)=c_{1} \lambda_{l}\left(g_{1}\right)+c_{2} l(l+d-2) \lambda_{l}\left(g_{2}\right)$.

Finally, we proceed to the remaining $d-1$ rows. We have

$$
\begin{aligned}
\int_{\mathcal{S}^{d-1}} M_{2}^{j k} \epsilon_{k} \mathrm{~d} \mathcal{S}_{\mathbf{w}} & =\frac{c_{1}}{\left|\hat{\mathbf{b}}_{j}(\mathbf{x})\right|} \int_{\mathcal{S}^{d-1}} \partial_{\eta_{j}}\left(g_{2}(\mathbf{x} \cdot \mathbf{w})\right) S^{l}(\mathbf{w}) \mathrm{d} \mathcal{S}_{\mathbf{w}}+ \\
& \frac{c_{2}}{\left|\hat{\mathbf{b}}_{j}(\mathbf{x})\right|} \int_{\mathcal{S}^{d-1}}\left[-g\left(R^{2}(1-\mathbf{x} \cdot \mathbf{w})\right)_{\eta_{k}^{\prime}}(\mathbf{x} \cdot \mathbf{w})_{\eta_{j}}-\right. \\
& \left.g\left(R^{2}(1-\mathbf{x} \cdot \mathbf{w})\right)(\mathbf{x} \cdot \mathbf{w})_{\eta_{j} \eta_{k}^{\prime}}\right] \frac{\partial^{\eta_{k}^{\prime}} S^{l}(\mathbf{w})}{\left|\hat{\mathbf{b}}_{k}(\mathbf{w})\right|^{2}} \mathrm{~d} \mathcal{S}_{\mathbf{w}}
\end{aligned}
$$

In the first term of the RHS of (45), as the integral is in the $\eta^{\prime}$ variables, we may pass the derivative through the integral to obtain

$$
\frac{c_{1}}{\left|\hat{\mathbf{b}}_{j}(\mathbf{x})\right|} \int_{\mathcal{S}^{d-1}} \partial_{\eta_{j}}\left(g_{2}(\mathbf{x} \cdot \mathbf{w})\right) S^{l}(\mathbf{w}) \mathrm{d} \mathcal{S}_{\mathbf{w}}=\frac{c_{1} \lambda_{l}\left(g_{2}\right) \partial_{\eta_{j}} S^{l}(\mathbf{x})}{\left|\hat{\mathbf{b}}_{j}(\mathbf{x})\right|}
$$

Integrating the second term of 45 by parts cancels the third term, leaving

$$
\begin{gathered}
\int_{\mathcal{S}^{d-1}} M_{2}^{j k} \epsilon_{k} \mathrm{~d} \mathcal{S}_{\mathbf{w}}=\frac{c_{1} \lambda_{l}\left(g_{2}\right) \partial_{\eta_{j}} S^{l}(\mathbf{x})}{\left|\hat{\mathbf{b}}_{j}(\mathbf{x})\right|}+ \\
\frac{c_{2}}{\left|\hat{\mathbf{b}}_{j}(\mathbf{x})\right|} \int_{\mathcal{S}^{d-1}} g\left(R^{2}(1-\mathbf{x} \cdot \mathbf{w})\right)(\mathbf{x} \cdot \mathbf{w})_{\eta_{j}} \partial_{\eta_{k}^{\prime}}\left(\frac{\partial^{\eta_{k}^{\prime}} S^{l}(\mathbf{w})}{\left|\hat{\mathbf{b}}_{k}(\mathbf{w})\right|^{2}} \mathrm{~d} \mathcal{S}_{\mathbf{w}}\right) \\
=\frac{c_{1} \lambda_{l}\left(g_{2}\right) \partial_{\eta_{j}} S^{l}(\mathbf{x})}{\left|\hat{\mathbf{b}}_{j}(\mathbf{x})\right|}-\frac{c_{2} l(l+d-2)}{\left|\hat{\mathbf{b}}_{j}(\mathbf{x})\right|} \int_{\mathcal{S}^{d-1}} g\left(R^{2}(1-\mathbf{x} \cdot \mathbf{w})\right)(\mathbf{x} \cdot \mathbf{w})_{\eta_{j}} S^{l}(\mathbf{w}) \mathrm{d} \mathcal{S}_{\mathbf{w}}
\end{gathered}
$$

using lemma 1 and the fact that $S^{l}$ is an eigenfunction. If we once again let $g_{3}(s)=\int_{0}^{R^{2}(1-s)} g(z) \mathrm{d} z$, we may pass the derivative through the integral as before to obtain

$$
\int_{\mathcal{S}^{d-1}} M_{2}^{j k} \epsilon_{k} \mathrm{~d} \mathcal{S}_{\mathbf{w}}=\left(c_{1} \lambda_{l}\left(g_{2}\right)+\frac{c_{2} l(l+d-2)}{R^{2}} \lambda_{l}\left(g_{3}\right)\right) \frac{\partial_{\eta_{j}} S^{l}(\mathbf{x})}{\left|\hat{\mathbf{b}}_{j}(\mathbf{x})\right|} .
$$

This establishes the claim in the remaining rows with $D^{22}\left(c_{1}, c_{2}, l\right)=c_{1} \lambda_{l}\left(g_{2}\right)+\frac{c_{2} l(l+d-2)}{R^{2}} \lambda_{l}\left(g_{3}\right)$. 
In summary, the continuous, $d$-dimensional eigenvalue problem (38) reduces to the $2 \times 2$ scalar eigenvalue problem

$$
\lambda\left(\begin{array}{l}
c_{1} \\
c_{2}
\end{array}\right)=\left(\begin{array}{cc}
\alpha+\lambda_{l}\left(g_{1}\right) & l(l+d-2) \lambda_{l}\left(g_{2}\right) \\
\lambda_{l}\left(g_{2}\right) & l(l+d-2) \frac{\lambda_{l}\left(g_{3}\right)}{R^{2}}
\end{array}\right)\left(\begin{array}{l}
c_{1} \\
c_{2}
\end{array}\right):=\Omega_{l}\left(\begin{array}{l}
c_{1} \\
c_{2}
\end{array}\right)
$$

where the $g_{i}$ remain as in the three dimensional case.

\section{$5 \quad$ Linear Stability and Linear Well-Posedness}

By reducing the linearized equations to a series of scalar problems, we can now readily identify the eigenvalues $\omega_{l}^{1,2}$ of the matrix $\Omega_{l}$. This then allows us to characterize the linear stability of sphere solutions: we need $\omega_{l}^{1,2}<0$ for all $l \geq 2$, together with $\omega_{0}^{1}<0, \omega_{0}^{2}=0, \omega_{1}^{1}<0$ and $\omega_{1}^{2}=0$ (rotation invariance manifests as a zero eigenvalue when $l=0$, translation invariance manifests as a zero eigenvalue for $l=1$ ). Therefore, the sphere is linearly stable if $\operatorname{tr}\left(\Omega_{l}\right)<0$ and $\operatorname{det}\left(\Omega_{l}\right)>0$ for all $l \geq 2$. Due to the form of $\Omega_{l}$, we see $\operatorname{det}\left(\Omega_{l}\right)>0$ can happen only if $\alpha+\lambda_{l}\left(g_{1}\right)$ and $\lambda_{l}\left(g_{3}\right)$ have the same sign. The condition $\operatorname{tr}\left(\Omega_{l}\right)$ then forces the negativity of both, so that we deduce the stability of mode $l$ occurs when

$$
\begin{aligned}
& \text { (i) } \alpha+\lambda_{l}\left(g_{1}\right)<0 \quad \text { (ii) } \lambda_{l}\left(g_{3}\right)<0 \\
& \text { (iii) }\left(\alpha+\lambda_{l}\left(g_{1}\right)\right) \lambda_{l}\left(g_{3}\right)>R^{2}\left[\lambda_{l}\left(g_{2}\right)\right]^{2} .
\end{aligned}
$$

This characterization of stability proves most useful in our analysis, and provides some additional insights. Indeed, from the relation for the perturbed density (35) and the perturbed radius (36), we see that a perturbation for which $c_{1}=0$ corresponds to a perturbation of density along the sphere, and not of the sphere itself. Condition (ii) therefore has a natural interpretation in terms of stability of the sphere with respect to perturbations of the density away from uniform. More specifically, as $\left(g_{3}\right)_{s}(s)=-R^{2} g\left(R^{2}(1-s)\right)$, the function $V(s):=-g_{3}\left(1-s / R^{2}\right)$ gives the potential that governs the pairwise interaction. We can then consider the potential energy $E\left(\rho_{S}\right)$ of the system as a function of the density,

$$
E\left(\rho_{S}\right)=\int_{\mathcal{S}^{2} \times \mathcal{S}^{2}} V\left(\frac{1}{2}|\mathbf{x}-\mathbf{w}|^{2}\right) \rho_{S}(\mathbf{x}) \rho_{S}(\mathbf{w}) \mathrm{d} \mathcal{S}_{\mathbf{x}} \mathrm{d} \mathcal{S}_{\mathbf{w}},
$$

and ask when $\rho_{S} \equiv 1$ corresponds to a minimum of 49 . Using the $\mathrm{F}-\mathrm{H}$ theorem as in 3.1 we find that this happens precisely when (ii) holds for all $l \geq 1$. Conversely, perturbations for which $c_{2}=0$ have no component tangential to the sphere. This happens when $l=0$ for instance. In this case (48) reduces to $\alpha+\lambda_{0}\left(g_{1}\right)<0$, which gives stability of the sphere with respect to pure dilations. Condition (i), then, enforces stability of the sphere with respect to purely normal modes.

Having characterized stability for finite $l$, we now wish to investigate the behavior of the spectrum as $l \rightarrow \infty$. The most classical question concerns the linear well-posedness of sphere solutions, i.e. when the eigenvalues remain bounded as $l \rightarrow \infty$. In practice, however, we primarily concern ourselves with a stronger notion: we wish to characterize when the sphere is eventually stable, that is, when $\omega_{l}^{1,2}<0$ for all $l$ sufficiently large. With eventually stable potentials we find that the finite number of unstable modes completely characterizes the ground state. We now address both issues using theorem 5.1, which furnishes asymptotic formulae for $\omega_{l}^{1,2}$ for sufficiently regular potentials.

To include the types of potentials that frequently occur in applications, we will assume $V \in C^{2}((0, \infty))$, but allow growth in $V$ as $s \rightarrow 0$. Specifically, we assume $V, V_{s}=o\left(s^{\frac{1-d}{2}}\right)$ as $s \rightarrow 0$. Additionally, to guarantee that $\lambda_{l}\left(g_{i}\right)$ are well-defined and to satisfy the hypothesis in the $\mathrm{F}-\mathrm{H}$ theorem, we must require $g_{i}(s)\left(1-s^{2}\right)^{\frac{d-3}{2}} \in L^{1}([-1,1])$. Recalling from 31] that

$g_{1}(s)=R^{2} g_{s}\left(R^{2}(1-s)\right)(1-s)^{2}-s g\left(R^{2}(1-s)\right), \quad g_{2}(s)=g\left(R^{2}(1-s)\right)(1-s), \quad\left(g_{3}\right)_{s}(s)=-R^{2} g\left(R^{2}(1-s)\right)$,

we should at least require $g_{s}\left(R^{2}(1-s)\right)(1-s)^{2}\left(1-s^{2}\right)^{\frac{d-3}{2}}, g\left(R^{2}(1-s)\right)\left(1-s^{2}\right)^{\frac{d-3}{2}} \in L^{1}$. We shall actually assume slightly more, namely that

$$
g_{s}\left(R^{2}(1-s)\right)(1-s)\left(1-s^{2}\right)^{\frac{d-3}{2}} \in L^{1}, g\left(R^{2}(1-s)\right)\left(1-s^{2}\right)^{\frac{d-3}{2}} \in L^{1} .
$$

Under these hypotheses, we have the following: 
Theorem 5.1. Let $V \in C^{2}((0, \infty))$ and $V, V_{s}=o\left(s^{\frac{1-d}{2}}\right)$ as $s \downarrow 0$. Assume also 50 . Then we have

(i) $\operatorname{tr}\left(\Omega_{l}\right) \sim \alpha$ and $\operatorname{det}\left(\Omega_{l}\right) \sim R^{2} l(l+d-2) \alpha \lambda_{l}\left(g_{3}\right)=o(1)$ as $l \rightarrow \infty$.

(ii) The sphere solution of radius $R$ given by $(32)$ is linearly well-posed, i.e. $\exists C$ such that $\omega_{l}^{1,2}<C$ for all $l \geq 0$.

(iii) Suppose $g(s)$ has a generalized power series expansion

$$
g(s)=\sum_{i=1}^{\infty} c_{i} s^{p_{i}}, \quad p_{1}<p_{2}<\cdots \quad \text { with } \quad c_{1}>0,
$$

that converges sufficiently rapidly. If the following conditions hold

$$
\text { (1) } \alpha<0 \text { and } \quad(2) p_{1} \in\left(-\frac{d-1}{2}, 0\right) \cup \bigcup_{n=0}^{\infty}(2 n+1,2 n+2),
$$

then the sphere is eventually stable i.e. (ii) holds for $C=0$.

Remark 5.2. The condition $\alpha<0$ enforces stability with respect to high-mode normal perturbations. Indeed, when $\alpha>0$ then all sufficiently high modes have a positive eigenvalue uniformly bounded away from zero, and the corresponding eigenfunction tends to a purely normal perturbation. Additionally, while the generalized power series (51) covers many potentials that occur in practice, such as the Morse potential, Gaussian potentials and power-law potentials, the conclusion of part (iii) holds for other classes of potentials without such an expansion. For instance, if the interaction kernel grows sufficiently rapidly near the origin while remaining smooth otherwise, part (iii) still holds. We conjecture (iii) still holds even if an expansion (51) only holds locally near zero, such as with the interaction (4).

Proof. To show (i), we need to estimate rate of decay of $\lambda_{l}\left(g_{i}\right)$. We recall that

$$
\lambda_{l}\left(g_{i}\right)=C(d) \int_{-1}^{1} g_{i}(s) P_{l, d}(s)\left(1-s^{2}\right)^{\frac{d-3}{2}} \mathrm{~d} s,
$$

where the constant $C(d)$ depends only on the dimension of space, $d$, and that the polynomial $P_{l, d}(s)$ satisfies the Gegenbauer equation

$$
\left(1-s^{2}\right) P_{l, d}^{\prime \prime}-(d-1) s P_{l, d}^{\prime}+l(l+d-2) P_{l, d}=0
$$

together with the normalization $P_{l, d}(1)=1$. From these definitions, we have that $f(s)\left(1-s^{2}\right)^{\frac{d-3}{2}} \in$ $L^{1}([-1,1])$ guarantees $\lambda_{l}(f)=o(1)$ (c.f. [14]).

Consider first $\lambda_{l}\left(g_{1}\right)$. The assumptions in 50 then suffice to have $g_{1}(s)\left(1-s^{2}\right)^{\frac{d-3}{2}} \in L^{1}$, so that $\lambda_{l}\left(g_{1}\right)=o(1)$. Proceeding now to $\lambda_{l}\left(g_{2}\right)$, we have,

$$
\begin{gathered}
l(l+d-2) \lambda_{l}\left(g_{2}\right)=\int_{-1}^{1} g_{2}(s)\left(1-s^{2}\right)^{\frac{d-3}{2}} l(l+d-2) P_{l}(s) \mathrm{d} s= \\
-\int_{-1}^{1} g_{2}(s)\left(1-s^{2}\right)^{\frac{d-1}{2}} P_{l, d}^{\prime \prime} \mathrm{d} s+(d-1) \int_{-1}^{1} s P_{l, d}^{\prime}(s)\left(1-s^{2}\right)^{\frac{d-3}{2}} g_{2}(s) \mathrm{d} s
\end{gathered}
$$

as $P_{l, d}$ satisfies the Gegenbauer equation (53). We integrate by parts in the first term, where the boundary terms vanish due to the growth assumption on $g=-V_{s}$. The identity $\left(s^{2}-1\right) P_{l, d}^{\prime}=l\left[s P_{l, d}(s)-P_{l-1, d}(s)\right]$ (equation (4.7.27) in [35]) then yields

$$
\begin{aligned}
l(l+d-2) \lambda_{l}\left(g_{2}\right) & =\int_{-1}^{1}\left(g_{2}\right)_{s}(s)\left(1-s^{2}\right)^{\frac{d-1}{2}} P_{l, d}^{\prime}(s) \mathrm{d} s \\
& =l \int_{-1}^{1}\left(g_{2}\right)_{s}(s)\left(1-s^{2}\right)^{\frac{d-3}{2}}\left[P_{l-1, d}(s)-s P_{l, d}(s)\right] \mathrm{d} s .
\end{aligned}
$$


As $\left(g_{2}\right)_{s}(s)=-R^{2} g_{s}\left(R^{2}(1-s)\right)(1-s)-g\left(R^{2}(1-s)\right)$, once again 50 suffices to have $(l+d-2) \lambda_{l}\left(g_{2}\right)=o(1)$, so that $\lambda_{l}\left(g_{2}\right)=o\left(l^{-1}\right)$. Similarly, for $\lambda_{l}\left(g_{3}\right)$ we compute

$$
\begin{gathered}
l(l+d-2) \lambda_{l}\left(g_{3}\right)=\int_{-1}^{1} g_{3}(s)\left(1-s^{2}\right)^{\frac{d-3}{2}} l(l+d-2) P_{l, d}(s) \mathrm{d} s \\
=-\int_{-1}^{1} g_{3}(s)\left(1-s^{2}\right)^{\frac{d-1}{2}} P_{l, d}^{\prime \prime} \mathrm{d} s+(d-1) \int_{-1}^{1} s P_{l, d}^{\prime}(s)\left(1-s^{2}\right)^{\frac{d-3}{2}} g_{3}(s) \mathrm{d} s .
\end{gathered}
$$

We integrate by parts twice, where the boundary terms vanish as before, and use that $\left(g_{3}\right)_{s}(s)=-R^{2} g\left(R^{2}(1-\right.$ $s)$ ) to discover

$$
\frac{1}{R^{2}} l(l+d-2) \lambda_{l}\left(g_{3}\right)=\int_{-1}^{1} \frac{\mathrm{d}}{\mathrm{d} s}\left\{g\left(R^{2}(1-s)\right)\left(1-s^{2}\right)^{\frac{d-1}{2}}\right\} P_{l, d}(s) \mathrm{d} s .
$$

Expanding the right hand side,

$$
\begin{aligned}
\frac{1}{R^{2}} l(l+d-2) \lambda_{l}\left(g_{3}\right) & =-\int_{-1}^{1}\left\{R^{2} g_{s}\left(R^{2}(1-s)\right)\left(1-s^{2}\right)+\right. \\
& \left.(d-1) s g\left(R^{2}(1-s)\right)\right\}\left(1-s^{2}\right)^{\frac{d-3}{2}} P_{l, d}(s) \mathrm{d} s .
\end{aligned}
$$

Again (50) allows us to conclude that the first and second terms vanish as $l \rightarrow \infty$. Therefore, $\lambda_{l}\left(g_{3}\right)=o\left(l^{-2}\right)$.

As $\lambda_{l}\left(g_{1}\right)=o(1), \lambda_{l}\left(g_{3}\right)=o\left(l^{-2}\right)$ and $\operatorname{tr}\left(\Omega_{l}\right)=\alpha+\lambda_{l}\left(g_{1}\right)+l(l+d-2) \lambda_{l}\left(g_{3}\right) R^{-2}$ from (46), we have $\operatorname{tr}\left(\Omega_{l}\right) \rightarrow \alpha$. As for $\operatorname{det}\left(\Omega_{l}\right)$, we have $\frac{\operatorname{det}\left(\Omega_{l}\right)}{R^{2} l(l+d-2)}=\alpha \lambda_{l}\left(g_{3}\right)+\lambda_{l}\left(g_{3}\right) \lambda_{l}\left(g_{1}\right)-\left[\lambda_{l}\left(g_{2}\right)\right]^{2}$. To discover the principal term, we note first that $\lambda_{l}\left(g_{3}\right) \lambda_{l}\left(g_{1}\right)$ clearly vanishes faster than $\alpha \lambda_{l}\left(g_{3}\right)$. Returning to $\lambda_{l}\left(g_{3}\right)$, we integrate by parts once and again use the identity $\left(s^{2}-1\right) P_{l, d}^{\prime}=l\left[s P_{l, d}(s)-P_{l-1, d}(s)\right]$ to find

$$
(l+d-2) \lambda_{l}\left(g_{3}\right)=\int_{-1}^{1} g\left(R^{2}(1-s)\right)\left(1-s^{2}\right)^{\frac{d-3}{2}}\left[s P_{l, d}(s)-P_{l-1, d}(s)\right] \mathrm{d} s .
$$

As $g_{2}(s)=g\left(R^{2}(1-s)\right)(1-s)$, this reads

$$
\begin{aligned}
& (l+d-2) \lambda_{l}\left(g_{3}\right)=-\lambda_{l}\left(g_{2}\right)-\lambda_{l-1}\left(g_{2}\right)+ \\
& \int_{-1}^{1} g\left(R^{2}(1-s)\right)\left(1-s^{2}\right)^{\frac{d-3}{2}}\left[P_{l, d}(s)-s P_{l-1, d}(s)\right] \mathrm{d} s .
\end{aligned}
$$

In particular, $\lambda_{l}\left(g_{3}\right)$ decays no faster than $\lambda_{l}\left(g_{2}\right) /(l+d-2)$. Since $\lambda_{l}\left(g_{2}\right)=o\left(l^{-1}\right),\left[\lambda_{l}\left(g_{2}\right)\right]^{2}$ decays faster. In other words,

$$
\frac{\operatorname{det}\left(\Omega_{l}\right)}{R^{2} l(l+d-2)}=\alpha \lambda_{l}\left(g_{3}\right)+\text { higher order terms. }
$$

This concludes the proof of (i) and (ii). To show (iii), note that $\left(g_{3}\right)_{s}(s)=-R^{2} g\left(R^{2}(1-s)\right)$. By lemma 5.3 shown below, we then obtain

$$
\lambda_{l}\left(g_{3}\right) \sim C\left(p_{1}, d, R\right) \frac{\sin \left(\pi p_{1}\right) c_{1}}{1+p_{1}} \Gamma\left(2+p_{1}\right) l^{-\left(2 p_{1}+d+1\right)} \quad \text { as } \quad l \rightarrow \infty
$$

where $C$ denotes a positive constant. Thus $\lambda_{l}\left(g_{3}\right)<0$ for sufficiently large $l$ as long as $p_{1}$ satisfies (52). In conjunction with $\alpha<0$ and (54), it then immediately follows $\operatorname{tr}\left(\Omega_{l}\right)<0$ and $\operatorname{det}\left(\Omega_{l}\right)>0$ for all $l$ sufficiently large, so the sphere is eventually stable. This concludes the proof.

Finally, we provide the lemma which was key in deriving part (iii) of Theorem 5.1 it will also prove useful in $\$ 6$ for constructing potentials with specified instabilities.

Lemma 5.3. Let $p+\frac{d-1}{2}>0$. Then we have

$$
\lambda_{l}\left((1-s)^{p}\right)=-2^{p+d-2} \frac{\operatorname{vol}\left(\mathcal{S}^{d-1}\right) \sin (\pi p) \Gamma\left(p+\frac{d-1}{2}\right) \Gamma(p+1) \Gamma\left(\frac{d-1}{2}\right) \Gamma(l-p)}{\pi \Gamma(l+p+d-1)},
$$




$$
\lambda_{l}\left((1-s)^{p}\right) \sim-\frac{2^{p+d-2}}{\pi} \operatorname{vol}\left(\mathcal{S}^{d-1}\right) \sin (\pi p) \Gamma\left(p+\frac{d-1}{2}\right) \Gamma(p+1) \Gamma\left(\frac{d-1}{2}\right) l^{-2 p-d+1}
$$

as $l \rightarrow \infty$.

Proof. Using the expression of Gegenbauer polynomials in terms of hypergeometric functions [35], we may write

$$
\begin{gathered}
\frac{\lambda_{l}\left((1-s)^{p}\right)}{\operatorname{vol}\left(\mathcal{S}^{d-1}\right)}=\int_{-1}^{1}(1-s)^{p+(d-3) / 2}(1+s)^{(d-3) / 2}{ }_{2} F_{1}\left(-l, l+d-2 ; \frac{d-1}{2} ; \frac{1-s}{2}\right) \mathrm{d} s \\
=2^{p+d-2} \int_{0}^{1} t^{p+(d-3) / 2}(1-t)^{(d-3) / 2}{ }_{2} F_{1}\left(-l, l+d-2 ; \frac{d-1}{2} ; t\right) \mathrm{d} t
\end{gathered}
$$

after the change of variables $t=\frac{1-s}{2}$. The generalized Euler transform for hypergeometric functions [33] then gives $\frac{\lambda_{l}\left((1-s)^{p}\right)}{\operatorname{vol}\left(\mathcal{S}^{d-1}\right)}=$

$$
2^{p+d-2} \mathrm{~B}\left(p+\frac{d-1}{2}, \frac{d-1}{2}\right){ }_{3} F_{2}\left(-l, l+d-2, p+\frac{d-1}{2} ; \frac{d-1}{2}, p+d-1 ; 1\right) .
$$

Using Saalschütz's theorem [5] to evaluate the hypergeometric term, we obtain

$$
\frac{\lambda_{l}\left((1-s)^{p}\right)}{\operatorname{vol}\left(\mathcal{S}^{d-1}\right)}=2^{p+d-2} \mathrm{~B}\left(p+\frac{d-1}{2}, \frac{d-1}{2}\right) \frac{\Gamma(l-p) \Gamma(p+d-1)}{\Gamma(-p) \Gamma(l+p+d-1)} .
$$

Using the identity $\Gamma(1-z) \Gamma(z)=\pi / \sin (\pi z)$ and expanding the beta function, we arrive at the stated expression,

$$
\pi \frac{\lambda_{l}\left((1-s)^{p}\right)}{\operatorname{vol}\left(\mathcal{S}^{d-1}\right)}=-2^{p+d-2} \sin (\pi p) \Gamma\left(p+\frac{d-1}{2}\right) \Gamma(p+1) \Gamma\left(\frac{d-1}{2}\right) \frac{\Gamma(l-p)}{\Gamma(l+p+d-1)} .
$$

For the asymptotics as $l \rightarrow \infty$, we note that Stirling's approximation gives $\Gamma(l-p) / \Gamma(l+p+d-1) \sim$ $l^{-2 p-d+1}$.

\section{$6 \quad$ Numerical Examples}

In this section, we provide numerical examples to illustrate how the different types of instability manifest as different qualitative behaviors of the ground states. We compute steady-state solutions to (2) for several different interactions $g$ using a fourth-order Adams-Bashforth scheme, with the number of particles $N=1000$. We take random initial conditions in all cases, and simulate until the $l_{\infty}$ norm of $(2)$ falls below .001/N .

We begin by considering a generalized Lennard-Jones interaction, $g(s)=s^{-p}-s^{-q}$ for $0<p, q<1$. To ensure a physically realistic potential consisting of short-range repulsion and far-field attraction, we must demand $p>q$. We then find, by $(52)$ (ii), that $\lambda_{l}\left(g_{3}\right)<0$ for all but finitely many $l$ with no further restriction. The sign of $\alpha$, then, completely determines the high-mode behavior. Direct computation shows that $\left(2 R^{2}\right)^{p-q}=\frac{2-q}{2-p}$, so that the condition $q<\frac{2 p-1}{2 p-2}$ determines when $\alpha<0$, and thus the eventual stability of the sphere. To illustrate both cases, we first select $(p, q)=\left(\frac{1}{3}, \frac{1}{6}\right)$ so that $\alpha<0$. By repeatedly using lemma 5.3 , we additionally verify that for all $l$ the stability conditions 48 hold. We therefore expect to accurately describe the solution as a sphere of radius $R=2^{-1 / 2}\left(\frac{11}{10}\right)^{3}$. Figure 2 (a) shows the resulting particle simulation. We next select $(p, q)=(1 / 2,1 / 4)$, so that $\alpha>0$ and we no longer expect the solution to concentrate on a co-dimension one manifold. As figure 2 (b) indicates, the solution instead fills a ball surrounding the origin; the color of a particle indicates its distance to the origin.

A more interesting picture begins to emerge when the sphere destabilizes yet remains eventually stable. As our examples will illustrate, the low mode instabilities fully describe the ground state. Due to the intricate steady-states it produces, cf. figure (1), we illustrate this phenomenon with the interaction $g(s)=$ $\frac{\tanh (a(1-\sqrt{2 s}))+b}{\sqrt{2 s}}$ introduced in [19]. The top of figure 3(a) depicts a computed steady-state of 2 with $(a, b)=(7,-.9)$. These parameters result in a mode $l=3$ instability. We then compute the eigenvector $\left(c_{1}, c_{2}\right)$ of $\Omega_{3}$, and use the result to construct the resulting surface from equation (36) and its corresponding 


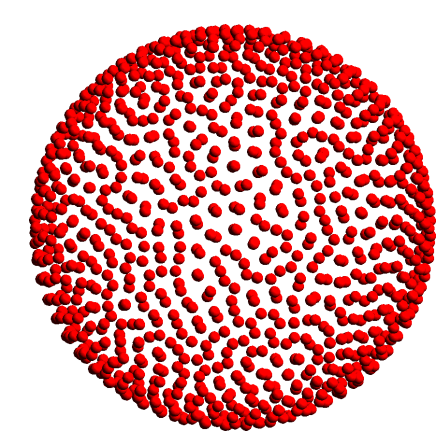

(a)

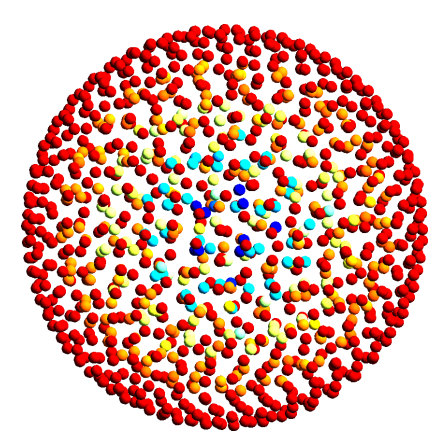

(b)

Figure 2: Equilibrium state of (2) with $N=1000$ particles and $g$ given by the generalized Lennard-Jones interaction $g(s)=s^{-p}-s^{-q}$. (a) $(p, q)=(1 / 3,1 / 6)$. Particles concentrate uniformly on a surface of the sphere, with no particles in the interior. (b) $(p, q)=(1 / 2,1 / 4)$. Particles fill the interior of a ball. The color-coding indicates the distance of a particle to their common center of mass.

density from equation (35), shown in the bottom of figure 3(a). This suggests that the linearized density echoes the overall shape of the full nonlinear problem in the vicinity of the bifurcation point. We perform the same computation for the parameter values $(a, b)=(9,0)$, which yields a mode $l=4$ instability, as shown in figure 3(b). Our theory and the experiments have excellent agreement.

The observation that particles align themselves with low-mode instabilities furnishes us with an avenue to construct potentials with intricate ground-states. Indeed, if we design an eventually stable interaction $g(s)$ with a single unstable mode, the resulting steady-state should resemble a spherical harmonic of that degree. To illustrate this procedure, we give an explicit construction of an interaction with a pure mode 5 instability.

First, we destabilize mode 5 . We accomplish this by enforcing $\lambda_{5}\left(g_{3}\right)>0$, which suffices due to the characterization (48). Using the identity

$$
s^{n}=\sum_{l=n, n-2, \ldots} \frac{(2 l+1) n !}{2^{\frac{n-l}{2}}(.5(n-l)) !(l+n+1) ! !} P_{l}(s),
$$

we can take $g_{3}(s)=\frac{s^{5}}{5}+p_{4}(s)$, where $p_{m}(s)$ denotes a polynomial of degree no more than $m$. Next, we choose $p_{3}(s)$ to stabilize modes $l \leq 4$. As $\lambda_{l}\left(p_{4}\right)=0$ whenever $l>4$, this choice does not affect the instability of mode 5. Indeed, taking $p_{3}=-3 s^{2}-4 s^{3}$ we obtain $R^{2} g\left(R^{2}(1-s)\right)=3 s^{2}+4 s^{3}-s^{4}$, where from (32) we see $R=1$. This yields an interaction $g(s)=3(1-s)^{2}+4(1-s)^{3}-(1-s)^{4}$ with a pure mode 5 instability, while all modes $l \geq 6$ contain exactly one zero eigenvalue. Lastly, we stabilize the remaining modes by adding a negative definite perturbation, $\tilde{g}(s)=g(s)+\epsilon f(s)$ for $f(s)=\frac{1}{\sqrt{s}}$. Using lemma 5.3 we find that

$$
R \lambda_{l}\left(f_{3}\right)=-\frac{2^{2+3 / 2}}{8 l^{3}+12 l^{2}-2 l-3}, \quad R \lambda_{l}\left(f_{2}\right)=\frac{\lambda_{l}\left(f_{3}\right)}{2}, \quad R \alpha_{f}=\frac{2^{5 / 2}}{3}, \quad R \lambda_{l}\left(f_{1}\right)=-2^{3 / 2} \frac{4 l^{2}+4 l-2}{8 l^{3}+12 l^{2}-2 l-3} .
$$

Therefore, for $\epsilon>0$ sufficiently small, $\tilde{g}$ has mode 5 unstable with all other modes stable. Figure 3(c) shows the resulting ground-state and spherical harmonic.

\section{Conclusion and Future Work}

In this paper, we addressed the fundamental question of understanding how the structure of particle potentials predict the types of patterns that can emerge. The potentials we focus on exhibit spherical geometry 

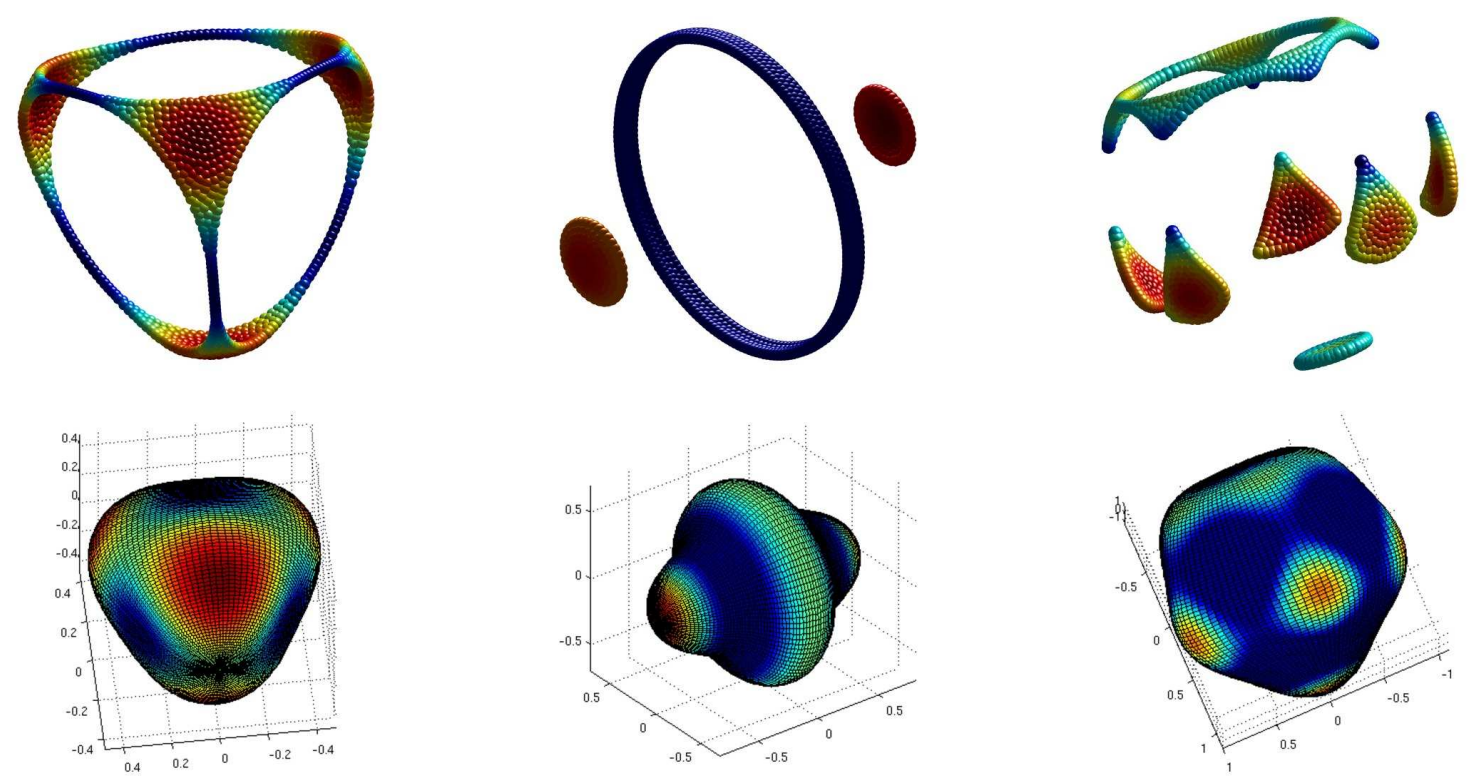

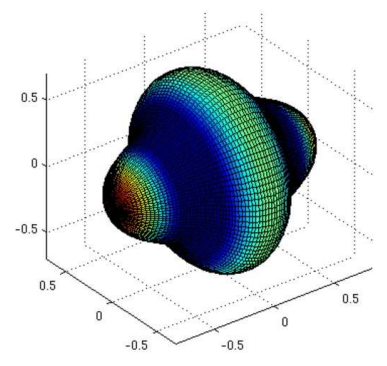

(b)

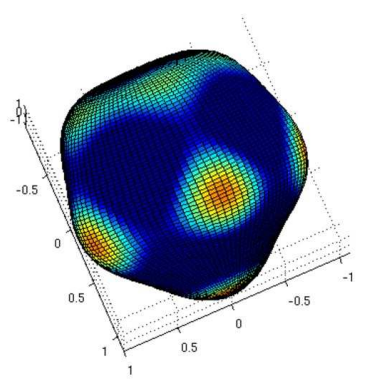

(c)

Figure 3: Top row: The result of the simulation of the gradient flow 2 with $N=1000$ particles for three different force laws. Bottom row: linearized solution corresponding to the instability mode as computed from (30). (a) Top: force law (4) with $(a, b)=(7,-.9)$. Bottom: Perturbation of a sphere using the spherical harmonic of mode $l=3, m=2$. (b) Top: Same as (a) but with $(a, b)=(9,0)$. Bottom: Perturbation of a sphere using the spherical harmonic of mode $l=4, m=0$. (c) Top: $g(s)=3(1-s)^{2}+4(1-s)^{3}-(1-$ $s)^{4}+\varepsilon s^{-1 / 2}$ with $\varepsilon=2^{-3 / 2}$. Sphere perturbed by a linear combination of the modes $l=5, m=5$ and $l=5, m=0$. The figures are color-coded according to the distance from the origin.

but solutions may form into both co-dimension one and co-dimension zero patterns. We analyzed this behavior by considering the linear stability of uniform sphere solutions to the equations governing a continuum of pairwise interacting particles on a surface. We reduced the $d$-dimensional eigenvalue problem into a decoupled series of $2 \times 2$ scalar problem involving a single spherical harmonic. This reduction allowed us to formulate stability and linear well-posedness conditions that have natural physical interpretations. These conditions provide us with a means to predict the co-dimension and the types of symmetries that will emerge in the resulting patterns. Using this predictive ability, we explicitly constructed a potential to yield a desired particle distribution, thereby addressing a particular case of the inverse statistical mechanics problem for self-assembly. In a subsequent paper, we extend this construction to arbitrary instabilities. Also, we note that our theory currently allows us to predict only the degree of a spherical harmonic that appears in the ground state. In dimensions $d \geq 3$, it remains an open issue to determine which spherical harmonic of a particular degree will arise. We plan to address this in future work.

\section{Acknowledgements}

We would like thank Andrew Bernoff for helpful conversations about this project. DU was partially funded by the UC President's Fellowship and NSF DMS-0902792. ALB acknowledges funding from NSF grants EFRI-1024765 and DMS-0907931, as well as ONR grant N000141010641. TK is supported by NSERC grant 47050 . 


\section{References}

[1] Eric L. Altschuler, Timothy J. Williams, Edward R. Ratner, Robert Tipton, Richard Stong, Farid Dowla, and Frederick Wooten. Possible global minimum lattice configurations for thomson's problem of charges on a sphere. Phys. Rev. Lett., 78(14):2681-2685, Apr 1997.

[2] Anna M. Barry, Glen R. Hall, and C. Eugene Wayne. Relative Equilibria of the $(1+N)$-Vortex Problem. arXiv, 1012.1002v1, 2010.

[3] A.J. Bernoff and C.M. Topaz. A primer of swarm equilibria. arXiv, 1008.0881v1, 2010.

[4] Michael P. Brenner, Peter Constantin, Leo P. Kadanoff, Alain Schenkel, and Shankar C. Venkataramani. Diffusion, attraction and collapse. Nonlinearity, 12(4):1071, 1999.

[5] J.L. Burchnall and A. Lakin. The theorems of Saalschütz and Dougall. Quart. J. Math., 2(1), 1950.

[6] Scott Camazine, Jean-Louis Deneubourg, Nigel R. Franks, James Sneyd, Guy Theraulaz, and Eric Bonabeau. Self-Organization in Biological Systems. Princeton Univ. Press, Princeton, 2003.

[7] Yao-Li Chuang, Y.R. Huang, M.R. D'Orsogna, and A.L. Bertozzi. Multi-vehicle flocking: Scalability of cooperative control algorithms using pairwise potentials. In Robotics and Automation, 2007 IEEE International Conference on, pages $2292-2299,2007$.

[8] Henry Cohn and Abhinav Kumar. Universally optimal distribution of points on spheres. J. Amer. Math. Soc., 20(1):99-148, 2007.

[9] Henry Cohn and Abhinav Kumar. Algorithmic design of self-assembling structures. PNAS, 106(24):9570-9575, 2009.

[10] Anna M. Delprato, Azadeh Samadani, A. Kudrolli, and L. S. Tsimring. Swarming ring patterns in bacterial colonies exposed to ultraviolet radiation. Phys. Rev. Lett., 87(15):158102, Sep 2001.

[11] M. R. D’Orsogna, Y. L. Chuang, A. L. Bertozzi, and L. S. Chayes. Self-propelled particles with soft-core interactions: Patterns, stability, and collapse. Phys. Rev. Lett., 96(10):104302, Mar 2006.

[12] Leah Edelstein-Keshet, James Watmough, and Daniel Grunbaum. Do travelling band solutions describe cohesive swarms? An investigation for migratory locusts. Journal of Mathematical Biology, 36:515-549, 1998. $10.1007 / \mathrm{s} 002850050112$.

[13] Szilard N. Fejer, Tim R. James, Javier Hernandez-Rojas, and David J. Wales. Energy landscapes for shells assembled from pentagonal and hexagonal pyramids. Phys. Chem. Chem. Phys., 11:2098-2104, 2009.

[14] George Gasper and Walter Trebels. A Riemann-Lebesgue lemma for Jacobi expansions. In A.I. Zayed M.E.H. Ismail, M.Z. Nashed and A.F. Gholeb, editors, Mathematical Analysis, Wavelets, and Signal Processing: an international conference on mathematical analysis and signal processes, volume 90 of Contemporary Mathematics. Amer. Math. Soc., Providence, R.I., 1995.

[15] N. R. Franks I. D. Couzin, J. Krauss and S. A. Levin. Effective leadership and decision-making in animal groups on the move. Nature, 433:513-516, 2005.

[16] Mohamed I. Jamaloodeen and Paul K. Newton. The $N$-vortex problem on a rotating sphere. II. heterogeneous platonic solid equilibria. Proc. R. Soc. A, 462(2075):3277-3299, 2008.

[17] S. A. Kaufmann. The Origins of Order: Self-Organization and Selection in Evolution. Oxford University Press, New York, 1933.

[18] Evelyn F. Keller and Lee A. Segel. Model for chemotaxis. Journal of Theoretical Biology, 30(2):225 $234,1971$. 
[19] Theodore Kolokolnikov, Hui Sun, David Uminsky, and Andrea L. Bertozzi. A theory of complex patterns arising from 2D particle interactions. Submitted.

[20] Robert Krasny. A study of singularity formation in a vortex sheet by the point-vortex approximation. J. Fluid Mech., 167:65-93, 1986.

[21] Andrew J. Leverentz, Chad M. Topaz, and Andrew J. Bernoff. Asymptotic dynamics of attractiverepulsive swarms. SIAM Journal on Applied Dynamical Systems, 8:880-908, 2009.

[22] Ryan Lukemana, Yue-Xian Lib, and Leah Edelstein-Keshet. Inferring individual rules from collective behavior. PNAS, 10(107), 2010.

[23] Andrew Majda and Andrea Bertozzi. Vorticity and Incompressible Flow. Cambridge University Press, 2002.

[24] A. Mogilner, L. Edelstein-Keshet, L. Bent, and A. Spiros. Mutual interactions, potentials, and individual distance in a social aggregation. Journal of Mathematical Biology, 47:353-389, 2003. 10.1007/s00285003-0209-7.

[25] Paul K. Newton and Takashi Sakajo. The $N$-vortex problem on a rotating sphere. III. ring configurations coupled to a background field. Proc. R. Soc. A, 463(2080):961-977, 2007.

[26] Paul K. Newton and Takashi Sakajo. Point vortex equilibria and optimal packings of circles on a sphere. Proc. R. Soc. A, 2010.

[27] J. K. Parrish and L. Edelstein-Keshet. Complexity, Pattern, and Evolutionary Trade-Offs in Animal Aggregation. Science, 284(99), 1999.

[28] A. Pérez-Garrido, M. J. W. Dodgson, and M. A. Moore. Influence of dislocations in thomson's problem. Phys. Rev. B, 56(7):3640-3643, Aug 1997.

[29] I. Prigogine. Order Out of Chaos. Bantam, New York, 1984.

[30] Mikael Rechtsman, Frank Stillinger, and Salvatore Torquato. Designed interaction potentials via inverse methods for self-assembly. Phys. Rev. E, 73(1):011406, Jan 2006.

[31] Mikael C. Rechtsman, Frank H. Stillinger, and Salvatore Torquato. Optimized interactions for targeted self-assembly: Application to a honeycomb lattice. Phys. Rev. Lett., 95(22):228301, Nov 2005.

[32] R.T. Seeley. Spherical harmonics. The American Mathematical Monthly, 73(4), 1966.

[33] L.J. Slater. Generalized hypergeometric functions. Cambridge University Press, 1966.

[34] Hui Sun, David Uminsky, and Andrea L. Bertozzi. A generalized Birkhoff-Rott equation for 2D active scalar problems. submitted, 2010.

[35] G. Szegö. Orthogonal Polynomials. Amer. Math. Soc., Providence, RI, 4th edition, 1975.

[36] Chad M. Topaz and Andrea L. Bertozzi. Swarming patterns in a two-dimensional kinematic model for biological groups. SIAM Journal on Applied Mathematics, 65(1):152-174, 2004.

[37] Salvatore Torquato. Inverse optimization techniques for targeted self-assembly. Soft Matter, 5:11571173, 2009.

[38] Lev Tsimring, Herbert Levine, Igor Aranson, Eshel Ben-Jacob, Inon Cohen, Ofer Shochet, and William N. Reynolds. Aggregation patterns in stressed bacteria. Phys. Rev. Lett., 75(9):1859-1862, Aug 1995.

[39] David J. Wales. Energy landscapes of clusters bound by short-ranged potentials. Chem. Eur. J. of Chem. Phys., 11(12):2491-2494. 
[40] McKay Hayley Wales, David J. and Eric L. Altschuler. Defect motifs for spherical topologies. Phys. Rev. B, 79(22):224115, Jun 2009.

[41] Wen Yang, A.L. Bertozzi, and Xiaofan Wang. Stability of a second order consensus algorithm with time delay. In Decision and Control, 2008. CDC 2008. 47th IEEE Conference on, pages 2926 -2931, 2008. 